\title{
Sharing a groundwater resource in a context of regime shifts
}

\author{
Julia de Frutos Cachorro* Katrin Erdlenbruch $^{\dagger} \quad$ Mabel Tidball $^{\ddagger}$
}

Article published in Environmental and Resource economics (2019)

DOI: $10.1007 / \mathrm{s} 10640-018-0233-0$

\begin{abstract}
We study the occurrence of shocks in a common groundwater resource problem using a differential game. In particular, we use Rubio and Casino's adaptation of the Gisser and Sánchez model where we introduce a sudden change in the dynamics of the resource, namely a decrease in the recharge rate of the aquifer. We compare the pareto optimal solution with open-loop and feedback equilibria. First, we show analytically how different solutions, at the steady state, depend on the intensity of the shock. Moreover, we show that the cost and the strategic effects are decreasing functions of the intensity of the shock, i.e. that all the solutions get closer at the steady state for more intense shocks. We finally apply the game to the particular case of the Western La Mancha aquifer. The aim of this application is to estimate how shocks influence the inefficiency of open loop and feedback strategies in terms of welfare. We show that this inefficiency decreases the earlier the shock occurs or the higher the intensity of the shock.
\end{abstract}

Keywords: Groundwater resource, differential game, strategic interactions, exogenous shock, regime shift.

\section{JEL: C72, Q25}

\footnotetext{
${ }^{*}$ University of Barcelona and BEAT, Barcelona, Spain.

${ }^{\dagger}$ Irstea, UMR G-EAU, University of Montpellier, Montpellier, France.

${ }^{\ddagger}$ INRA, UMR Lameta, Montpellier, France.
} 


\section{Introduction}

In this paper, we study the exploitation of a common groundwater resource as a differential game in order to take into account the strategic and dynamic interactions between the users of the resource. Specifically, we consider a groundwater resource used for irrigation by several farmers. Common groundwater resources are often exploited under a common property regime, that is the access is restricted to land owners situated over the aquifer. Numerous papers have studied this issue (for example Gisser and Sánchez (1980) [4], Negri (1989) [8], Provencher and Burt (1993) [11], Rubio and Casino (2001) [12]) and have concluded that private exploitation is inefficient, in terms of stock and welfare, in comparison to optimal exploitation.

This inefficiency is due to the various externalities which appear because of the sharing of this type of resource, namely the pumping cost externality which characterizes the fact that withdrawals made by one farmer lower the water-table level, resulting in an increase in pumping costs for the other users. On the other hand, the stock externality, also called strategic externality, represents competition between farmers because of the limited availability of the water stock (see Provencher and Burt (1993) [11]).

Gisser and Sánchez (1980) [4] showed the inefficiency of private exploitation for the Pecos River Basin, New Mexico. They also characterized the analytical difference between the optimal and private solutions, and they concluded that the difference is negligible if the capacity of the aquifer is large. Nieswiadomy (1988) [9] called this consideration the Gisser and Sánchez effect (GSE), see Koundouri (2004) [5] for an overview. The most important policy implication derived from this study is that regulation of a common groundwater resource is not justified if the difference of welfare from private and optimal exploitations is insufficiently important. However, authors assume that farmers behave myopically in the calculation of the private solution, that is, farmers take decisions over a short period of time, without considering the impact of the other users on the available stock.

Several studies have used game theory to take into account the strategic and dynamic interactions between the resource users when computing the private solution (for example Negri (1989) [8], Provencher and Burt (1993) [11], Rubio and Casino (2001) [12]). In [8], 
Negri characterizes analytical solutions of the water-table level at the steady state for two types of Nash equilibria, open-loop and feedback solutions, and for the socially optimal case, also referred as pareto optimal case in the literature. He shows that the difference between the socially optimal solution and the open-loop solution is positive and captures the pumping cost externality. Moreover, he shows that the difference between the open-loop solution and the feedback solution is also positive and captures the strategic externality. The difference between the socially optimal and the feedback solutions is then positive and represents the inefficiency of private exploitation. Provencher and Burt [11] take up Negri's ideas to prove, in a general way, that if the objective function of the problem is concave, the feedback solution is inefficient, in comparison with the socially optimal solution. In [12], Rubio and Casino adapt the Gisser and Sánchez model as a differential game and derive analytical solutions of the socially optimal, open loop and feedback cases over an infinite planning horizon. They also confirm Negri's result: strategic behaviour exacerbates the inefficiency of private solutions. Moreover, they confirm the Gisser-Sánchez rule when the strategic externality is considered: for large aquifers, the different solutions get closer at the steady state.

The motivation of our work is based on the idea that some exogenous threats are not taken into account in previous studies, as for example the occurrence of regime shifts. We focus on the study of the inefficiency of the private solution with strategic and dynamic interactions and in presence of an anticipated shock. More specifically, we take Rubio and Casino's game in [12] and we introduce a sudden change in the dynamics of the resource, which leads to an abrupt decrease of the water availability for the users of the resource. We model this shock as a decrease in the recharge of the aquifer. More generally, such a shock corresponds to a regime shift. We then compare the pareto optimal solution with open-loop and feedback Nash equilibria.

In [1], de Frutos Cachorro et al. (2014) study how information about this type of shock affects the optimal management of the water resource by a centralized regulator (a water agency). For the deterministic case, when the date of the shock is known, the water agency would prepare for the event by applying an incautious extraction strategy before the occurrence of the shock. Such a result can already be found in the literature 
dealing with the impact of irreversible events (see Tsur and Zemel (2014) [15]), where the phenomenon is known as the "impatience effect".

In this paper, we combine Rubio and Casino's game theory approach and de Frutos Cachorro et al. study on the effect of regime shifts, in order to assess the difference between the pareto optimal and private solutions with strategic and dynamic interactions between users.

We show that the combined effect of strategic interactions and this type of shock leads to an overexploitation of the resource in the short, medium and long run. Moreover, we study the inefficiency of private exploitation with respect to the intensity and date of occurrence of the shock. From an economic point of view, we could expect that the higher the intensity of the shock, i.e. the lower the quantity of water available, the higher the overall pumping costs and the competition between users. However, we show that cost and strategic effects are particularly important for low-intense or later occurring shocks. Finally, we estimate the inefficiency of private exploitation in terms of welfare for a particular case, the Western la Mancha aquifer. This aquifer is situated in the South of Spain, under a semi-arid climate where dry periods are frequent. Moreover, in the last decades, the aquifer has suffered from various inefficient regimes of exploitation. Our results suggest that some regulation of the aquifer is justified. Indeed, although efficiency gains from following the pareto optimal solution are lower than in absence of shocks, they still reach several millions of Euros, for example 37 millions of Euros in the situation of a mid-intensity shock. The pareto optimal solution could be implemented by imposing licenses, such that extractions over time correspond to the pareto optimal extractions.

This paper is organized in the following way. In section 2, we present Rubio and Casino's game and introduce the case of an exogenous and deterministic shock therein. In section 3, we describe analytical resolutions of the problem for different information structures. In section 4 , we compute the pareto optimal solution corresponding to the problem. In section 5, we first compare the different analytical solutions, in terms of long-term stocks. We then make a numerical application of the model to the Western La Mancha aquifer with the aim to estimate, in terms of stocks and welfare, the inefficiency of private solutions. Finally, in section 6 , we conclude and give some perspectives for future 
research.

\section{The model}

First, we present the adaptation of the Gisser and Sánchez model (1980) [4] as a differential game developed by Rubio and Casino $(2001,2003)$ ([12], [13]). In [4], the demand for irrigation water is a linear function,

$$
g=a-b p, \quad a, b>0,
$$

where $g$ represents water pumping, $p$, the price of water and $a$ and $b$ are parameters.

In [12], Rubio and Casino assume that the number of farmers, $M$, is fixed and finite over time. The individual demand for irrigation water can be described as a linear function,

$$
g_{i}=\theta_{i}(a-b p), \quad i=1 . . M,
$$

where $0<\theta_{i}<1$ and $\sum_{i=1}^{M} \theta_{i}=1$. Thus,

$$
\sum_{i=1}^{M} g_{i}=\sum_{i=1}^{M} \theta_{i}(a-b p)=a-b p=g .
$$

Moreover, the revenue of the farmer $i$ is equal to

$$
\int_{g_{i}} p(x) d x=\int_{g_{i}} \frac{a-\frac{g_{i}}{\theta_{i}}}{b} d x=\frac{a}{b} g_{i}-\frac{1}{2 b \theta_{i}} g_{i}^{2} .
$$

We assume that the marginal cost of extraction is a linear function that depends on $G$, the stock of the aquifer. Total costs of extraction are then

$$
\bar{C}=(z-c G) g, \quad z, c>0,
$$

where $z$ is the sum of fixed costs and the maximum marginal cost of extraction and $c$ the slope of the marginal pumping cost function. As $z$ and $c$ do not depend on the rate of extraction, the individual pumping cost of the $i^{\text {th }}$ farmer is

$$
\bar{C}_{i}=(z-c G) g_{i}, \quad z, c>0 .
$$


The dynamics of the aquifer can be described as

$$
\dot{G}=-(1-\alpha) g+r=-(1-\alpha) \sum_{i=1}^{M} g_{i}+r,
$$

where $r$ is the recharge rate and $\alpha$ the return coefficient, $\alpha \in[0,1)$.

Assuming that interactions between farmers are rational and non-cooperative, the problem of the $i^{\text {th }}$ farmer is to maximize individual welfare, defined as the present value of his future profits, where $\rho$ is the discount rate, taking into account the dynamics of the aquifer (equation (6)) and given initial conditions and positivity constraints:

$$
\max _{g_{i}(.)} \int_{0}^{\infty} \quad F_{i}\left(G, g_{i}\right) e^{-\rho t} d t
$$

where,

$$
\begin{gathered}
F_{i}\left(G, g_{i}\right)=\frac{a}{b} g_{i}-\frac{1}{2 b \theta_{i}} g_{i}^{2}-(z-c G) g_{i}, \\
\dot{G}=-(1-\alpha) \sum_{i=1}^{M} g_{i}+r, \\
G(0)=G_{0} \quad \text { given, } \\
g_{i} \geq 0 \quad G \geq 0 .
\end{gathered}
$$

Now, we disturb the system of the resource by introducing an exogenous and deterministic shock in the dynamics of the aquifer, as proposed by de Frutos Cachorro et al. (2014) [1]. This disturbance represents a sudden reduction on the recharge rate, $r$, at time $t_{a}$, known to the users. Thus, from $t_{a}$ on, the recharge rate switches from $r=r_{1}$ to $r=r_{2}$, with $r_{1}>r_{2}$. This can happen because of an exceptional extraction of groundwater for other uses from $t_{a}$ on, as for example the construction of a reservoir or a transfer to another river basin.

The problem of the $i^{t h}$ farmer becomes then (7), constrained by the dynamics: 


$$
\dot{G}=\left\{\begin{array}{lll}
-(1-\alpha) \sum_{i=1}^{M} g_{i}+r_{1} & \text { if } & t \leq t_{a} \\
-(1-\alpha) \sum_{i=1}^{M} g_{i}+r_{2} & \text { if } & t>t_{a}
\end{array}\right.
$$

with $r_{1}>r_{2}, F_{i}\left(G, g_{i}\right)$ from equation (8), and conditions (10) and (11).

In what follows, we are going to solve the game including this shock when players have different information structures: open-loop (OL) and feedback (FB), as in Rubio and Casino $(2001,2003)$ ([12], [13]). In every case, we solve problems in two steps: between $t_{a}$ and $\infty$ and between 0 and $t_{a}$. We anticipate that equilibria of the various problems will be different according to the structure of information used by players. We call adaptation behavior the extraction decisions that are implemented by farmers from $\mathrm{t}=0$ until the end of the planning horizon, knowing from $\mathrm{t}=0$ that a shock will occur at some fixed $t_{a}{ }^{1}$.

\section{Non-cooperative cases}

\subsection{Resolution of the open-loop case}

We assume that farmers made a commitment at the initial instant $(t=0)$ about their path of extractions over time. This is an open-loop information structure. The Hamiltonian corresponding to the problem of the ith farmer is:

$$
H_{i}=\left\{\begin{array}{lll}
F_{i}\left(G, g_{i}\right)+\pi_{i}(t)\left(r_{1}-(1-\alpha) \sum_{i=1}^{M} g_{i}\right) & \text { if } & t \leq t_{a} \\
F_{i}\left(G, g_{i}\right)+\pi_{i}(t)\left(r_{2}-(1-\alpha) \sum_{i=1}^{M} g_{i}\right) & \text { if } & t>t_{a}
\end{array}\right.
$$

with $F_{i}\left(G, g_{i}\right)$ from equation (8), and $\pi_{i}(t)$, the adjoint variable. $G(t)$ and $\pi_{i}(t)$ are continuous functions in the interval $[0, \infty)$. We have detailed the analytical resolution of the open-loop game in appendix A.

The open-loop solution supposes that the farmer does not change his extraction decisions over the whole time horizon. This assumption is not very realistic whenever the farmer can observe the resource stock, and then indirectly the actions of the other users of the resource. If he acts strategically he might want to adapt to the other users' actions.

\footnotetext{
${ }^{1}$ On the other hand, we call non-adaptation behaviour, when farmers do not have information about the shock until it happens and then change their extraction decisions just from the date of occurrence.
} 
As discussed in Negri [8], the open-loop solution does not allow to account for strategic interactions among resource users, but the feedback solution does.

\subsection{Resolution of the feedback case}

In the feedback information structure, farmers observe the level of the resource during the planning period, i.e. they have information about the state (or the water-table level) of the resource over time. Indeed, in many cases of groundwater management, the water-table level can be observed from the individual wells. Thus, it is more credible for the farmers to maximize their profit assuming that actions or strategies made by the other farmers depend not only on time but on the state of the groundwater resource. We are going to solve this case on the basis of the principle of dynamic programming. The full resolution of the problem is detailed in Appendix B.

\section{The pareto optimum}

One of the objectives of this paper being to estimate the inefficiency of various equilibria defined previously, we need to define the efficient solution of the problem: the pareto optimum. The difference between the pareto optimal solution and any non-cooperative solution will define inefficiency, either in terms of stocks or in terms of welfare. Inefficiency in terms of stocks is measured comparing steady state levels of stocks, while inefficiency in terms of welfare is measured comparing gains obtained over the whole time horizon.

For the pareto optimal solution, we suppose that a social planner decides how to manage the resource. The problem for the regulator is to maximize the social welfare, defined as the present value of the sum of future revenues of the $M$ users of the resource.

The problem for the regulator is:

$$
\max _{\left\{g_{i}\right\}_{i=1}^{M}} \int_{0}^{\infty} \sum_{i=1}^{M} F_{i}\left(G, g_{i}\right) e^{-\rho t} d t,
$$

with $F_{i}\left(G, g_{i}\right)$ described in equation (8), constrained by equation of motion (9), initial condition (10) and positivity conditions (11). 
Now, if a shock occurs at the known date $t_{a}$, the problem for the social planner becomes (14), constrained by the equation of motion (12), where $r_{1}$ and $r_{2}$ are values of the recharge rate before and after $t_{a}$, respectively, with initial and positivity conditions described in equations (10) and (11). The full resolution of this problem is detailed in Appendix C. As discussed in de Frutos Cachorro et al. (2014) [1], optimal adaptation behavior in presence of such shocks is characterized by a more intense extraction behavior in the short run (before the occurrence of the shock), namely "the impatience effect", and a less intense extraction behavior in the long run, compared to the situation without shocks. This is because the presence of a shock reduces the recharge rate, i.e. the water available in the future, thus reducing extraction possibilities in the long run. In addition, the "impatience effect" induces farmers to accelerate extraction in the short run to offset future losses in the long run. One important issue is now to see whether this compensating short-term behavior might be changed in case of non-cooperative solutions.

In what follows, we analyze and compare the pareto optimal solution with the different equilibria (open-loop and feedback) obtained when such a shock takes place.

\section{$5 \quad$ Results}

\subsection{Theoretical Results}

In this section, we compare analytically the efficiency of the different stock solutions at the steady state. We remind that inefficiency in terms of stocks is defined as the difference between steady-state stock levels obtained from the pareto optimal solution and different private equilibria. From equations $(67),(30),(52)$ in the Appendices, we obtain solutions of the stock for the pareto optimum (PO), the open-loop (OL) and the feedback case (FB), with $\mathrm{M}$, the number of symmetric farmers $(M>1)$ :

$$
G_{\infty}^{P O}=\frac{r_{2}}{c b(1-\alpha)}+\frac{r_{2}}{\rho}-\frac{a}{c b}+\frac{z}{c}
$$




$$
G_{\infty}^{O L}=\frac{r_{2}}{c b(1-\alpha)}+\frac{r_{2}}{M \rho}-\frac{a}{c b}+\frac{z}{c}
$$

and

$$
G_{\infty}^{F B}=\frac{r_{2}}{M(1-\alpha) a_{1}^{*}}-\frac{b_{1}^{*}}{a_{1}^{*}}
$$

with expressions $b_{1}^{*}=b_{1}^{*}\left(r_{2}\right)<0, a_{1}^{*}>0$ and

$$
\lim _{r_{2} \rightarrow 0} G_{\infty}^{F B}=\lim _{r_{2} \rightarrow 0} G_{\infty}^{O L}=\lim _{r_{2} \rightarrow 0} G_{\infty}^{P O}=\frac{-a+z b}{c b}>0 .
$$

See Appendix D for more details about the positivity of the steady states and the proofs of propositions of this section.

Moreover, solutions of the pumping rate at the steady state are:

$$
g_{\infty}^{F B}=g_{\infty}^{O L}=g_{\infty}^{P O}=\frac{r_{2}}{(1-\alpha) M} .
$$

Proposition 1 When the value of the recharge rate upon occurrence of the shock, $r_{2}$, decreases (respectively increases), the level of the stock at the steady state decreases (respectively increases) for the different cases ( $P O, O L$ and $F B$ ) and reaches the same limit value when $r_{2}$ goes to zero. Moreover, solutions of pumping rates at the steady state are the same for the different information structures and decrease (respectively increase), the lesser (respectively the greater) the value of $r_{2}$.

Hence, the shock decreases steady state stock levels for both the pareto optimal solution and the non-cooperative solutions. Negri shows in [8] that open-loop solutions, and in a more important way feedback solutions, are inefficient with respect to the pareto optimal solution. We have now to study the difference of the various solutions to further quantify the effect of the shock on the different pumping strategies, or more specifically, to analyze if the ranking of efficiency of solutions is maintained in presence of such a shock. Differences are calculated and described below. The difference,

$$
G_{\infty}^{P O}-G_{\infty}^{O L}=\frac{r_{2}}{\rho}\left(1-\frac{1}{M}\right)
$$


captures the pumping cost effect, while the difference

$$
G_{\infty}^{O L}-G_{\infty}^{F B}=r_{2}\left(\frac{1}{M \rho}+\frac{1}{(1-\alpha) c b}-\frac{1}{M(1-\alpha) a_{1}^{*}}\right)-\frac{a}{c b}+\frac{z}{c}+\frac{b_{1}^{*}}{a_{1}^{*}},
$$

captures the strategic (or stock) effect.

Proposition 2 When a deterministic shock on the recharge rate takes place, the cost and strategic effects are positive.

Proposition 2 shows that the cost and strategic effects are positive when there is a shift on the recharge rate at a given date. This means that both non-cooperative solutions are inefficient, in terms of stocks, in presence of the shock. An important issue is now to study how these inefficiencies are influenced by a change in the value of the shock.

Proposition 3 When $r_{2}$ decreases (respectively increases), the cost and strategic effects decrease (respectively increase).

Proposition 3 exposes one of our main results: it shows that pumping strategies, at the steady state, derived from private and optimal solutions get closer if the aquifer recharge decreases. This result might be counter-intuitive at first sight as the occurrence of a shock reduces the inefficiency of the different non-cooperative solutions. It may be explained by the fact that a reduction on the recharge rate, implies a reduction on the water available to share in the future. Thus, inefficiency cannot develop to the same extent as in a case with greater water availability.

We remind that Rubio and Casino found the same expressions (19) and (20) in [12] in their model without shock. They show first that the difference between solutions declines as the discount rate and/or the number of farmers increases. They also confirm that the same result is obtained when the storage capacity of the aquifer ${ }^{2}$ increases (GSE effect). In this paper, we add to Rubio and Casino's result the importance of a recharge rate variation.

In order to correct this inefficiency, instruments such as licences or quotas can be used that bring the resource user to respect pareto optimal extraction paths. Let's consider

\footnotetext{
${ }^{2}$ In [12], the storage capacity of the aquifer corresponds to the expression $G / H$, where $\mathrm{G}$ is the volume of water and $\mathrm{H}$ is the water-table height of the aquifer.
} 
a non-transferable quota depending on the resource stock and take the example of the feedback case.

Proposition 4 There exists an optimal quota $\bar{g}(G)$ with $\bar{g}=g^{P O}$, such that $g=g^{P O}$ is a solution of the feedback problem, constraint by $g \leq \bar{g}$.

Proposition 4 shows that an optimal quota $\bar{g}(G)$ could be imposed in order to reach the pareto optimal solution, and this quota corresponds to the pareto optimal extraction path.

However, Gisser and Sanchez argued in [4] that regulation of a common groundwater resource is not justified if the difference of welfare from private and optimal exploitations is insufficiently important. In what follows, we apply our game to the Western la Mancha aquifer in order to estimate inefficiency in terms of welfare ${ }^{3}$ and not only in terms of stocks. This application also allows us to estimate the magnitude of inefficiencies, for shocks of different intensities and occurring at different dates.

\subsection{Numerical application}

In this section, we use parameter values of the Western La Mancha aquifer (WLM) from de Frutos Cachorro et al. (2014) [1], Esteban and Albiac (2011) [2] and Esteban and Dinar (2012) [3]. The parameter values used are listed in Table 1.

Covering a surface area of $5500 \mathrm{~km}^{2}$ in the South of Spain, the WLM aquifer is located in a semi-arid region characterized by low and irregular rainfall and by high evapotranspiration due to the significant number of sunny days (cf. López Sanz (1993) [7]). These conditions give rise to a very low efficient recharge rate of the aquifer of around $20 \%$ of precipitation. Moreover, drought episodes can be sustained over time, lasting several years, as happened in 1980-1985 and 1990-1996 (cf. Olcina Cantos (2001) [10]). In the last decades, the WLM aquifer witnessed a critical decrease in water-table levels, due to

\footnotetext{
${ }^{3}$ We remind that inefficiency in terms of welfare is defined as the difference between gains obtained from the pareto optimal solution and the different private equilibria over the whole time horizon.
} 
the development of intensive irrigated agriculture, which increased groundwater extraction ( 3 thousand million $\mathrm{m}^{3}$ over the past three decades according to López-Gunn (2012) [6]), coupled with inefficient management regimes (Esteban and Albiac (2011) [2]). The degradation of water-table levels has caused damage to aquatic ecosystems as the "Mancha Húmeda" Biosphere reserve and also affects human uses downstream.

\begin{tabular}{cllr} 
Parameters & Description & Units & Value \\
\hline b & Water demand slope & (Million Cubic Meters/Year) ${ }^{2}$ Euros $^{-1}$ & 0.097 \\
a & Water demand intercept & Million Cubic Meters/Year & 4403.3 \\
z & Pumping costs intercept & Euros/Million Cubic Meters & 266000 \\
c & Pumping costs slope & Euros/(Million Cubic Meters) & 3.162 \\
r & Natural recharge & Million Cubic Meters/Year & 360 \\
$G_{0}$ & Stock level (in volume) & Million Cubic Meters & 80960 \\
$H_{0}$ & Current water table & Meters & 640 \\
$S_{L}$ & Surface elevation & Meters & 665 \\
$\mathrm{~A}$ & Aquifer area & Square Kilometers & 5500 \\
$\mathrm{~S}$ & Storativity coefficient & unitless & 0.023 \\
$\rho$ & Social discount rate & Year & \\
$\alpha$ & Return flow coefficient & unitless & 0.05 \\
$\mathrm{M}$ & Number of players & unitless & 0.2 \\
\hline
\end{tabular}

Table 1: Values of parameters of the Western La Mancha aquifer.

In what follows, we first estimate the inefficiency, as defined in the previous section, in terms of stocks, and then in terms of welfare. We also discuss the profitability of the different solutions until the date of the shock, that is gains obtained in $\left[0, t_{a}\right]$. Moreover, we complete this analysis by studying the problem when the intensity and the date of occurrence of the shock vary.

In Figure 1, we observe optimal solutions of stock $G^{*}(t)$ (on the left) and pumping rate $g^{*}(t)$ (on the right), in particular the pareto optimal (PO) (in green), the open loop 

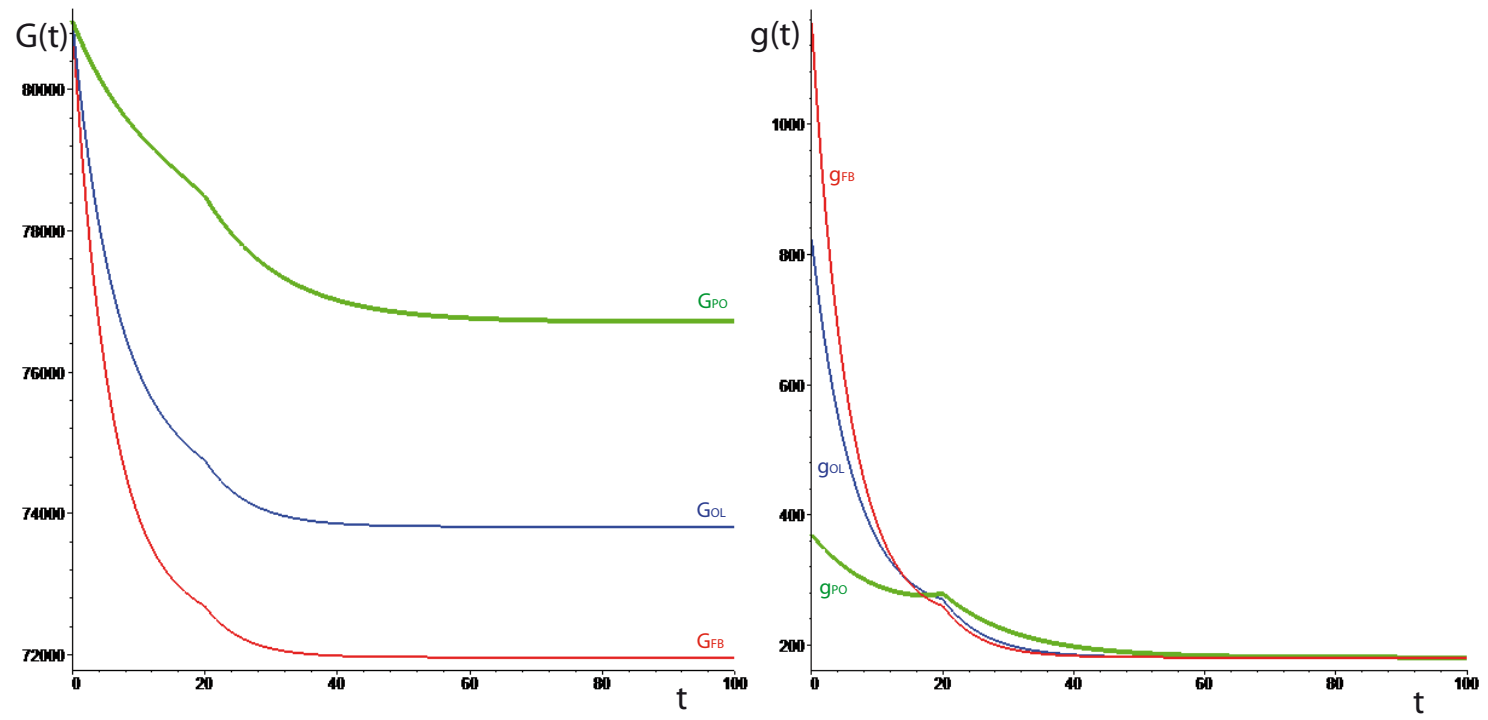

Figure 1: Solutions of $G^{*}(t)$ (left-hand side) in millions of cubic meters and $g^{*}(t)$ (righthand side) in millions of cubic meters per year : the pareto optimum (in green), the open-loop (in blue) and the feedback (in red) cases, when $r_{1}-r_{2}=70$ and $t_{a}=20$ years.

(OL) (in blue) and the feedback solutions (FB) (in red), for a shock of mid-intensity of 70 millions of cubic meters per year $\left(\mathrm{Mm}^{3} /\right.$ year $)$, i.e. $r_{1}=360$ and $r_{2}=290$ at the $20^{\text {th }}$ year of exploitation of the aquifer $\left(t_{a}=20\right.$ years $)$. In what follows, we use this shock as a baseline case. $^{4}$

Focusing on the left-hand side of the figure, we note that $G^{P O}(t)>G^{O L}(t)>G^{F B}(t)$ for all $t$. This gives the ranking of efficiency in terms of stocks. In particular at the steady state, the pareto optimal stock reaches a level of $76711 \mathrm{Mm}^{3}$, which is higher than levels obtained by the OL and FB solutions (of around 73811 and $71962 \mathrm{Mm}^{3}$ respectively). Thus, the difference between the pareto optimal and the open-loop solutions is $2899 \mathrm{Mm}^{3}$ whereas the difference between the pareto optimal and the feedback solutions is $4749 \mathrm{Mm}^{3}$. We hence confirm theoretical results proved in Proposition 2: the cost and strategic effects in the steady state remain positive when a shock takes place. Moreover, we observe on

\footnotetext{
${ }^{4}$ This choice is motivated by the fact that the decrease of the water-table obtained in $t_{a}=20$ can be compared to the estimated drop of $3000 \mathrm{Mm}^{3}$ over the last 30 years reported by López-Gunn.
} 
the right-hand side that the pumping rate at the steady state is the same for the different solutions, with a value of approximately $181 \mathrm{Mm}^{3} /$ year, as demonstrated in Proposition 1.

We can also analyze the problem before the occurrence of the shock, between $\mathrm{t}=0$ and $t=t_{a}=20$ years. On the right hand, we note that total extractions until the arrival of the shock, measured by the areas under the curves in $\left[0, t_{a}\right]$, are higher in the feedback case $(9$ $\left.672 \mathrm{Mm}^{3}\right)$ than in the OL $\left(8383 \mathrm{Mm}^{3}\right)$ and PO $\left(6044 \mathrm{Mm}^{3}\right)$ solutions. This means that the feedback strategy is the less conservative for the resource until $t_{a}=20$ years. In other words, the "impatience effect", that is the increase of extractions before the occurrence of the shock is most important in the feedback case, and least important in the pareto optimal solution.

Let us now calculate the inefficiency in terms of welfare of the private solutions ${ }^{5}$ over the whole time horizon (in $[0, \infty)$ ), as well as their profitability before the occurrence of the shock (in $\left[0, t_{a}\right]$ ). Over the whole time horizon, the inefficiency of the FB solution with regard to the PO solution is estimated at 37478 thousands of Euros and the inefficiency of the OL solution with respect to the PO solution at 14393 thousands of Euros. This can be seen in Table 2, column 3. Maybe more surprisingly, in $\left[0, t_{a}\right]$, the difference of welfare between the $\mathrm{PO}$ and FB solutions is positive, but the difference between the PO and OL solutions is negative, that is the OL strategy is more profitable than the PO solution until the occurrence of the shock, (as can be seen in Table 2 column 2). However, this result is not related to the occurrence of the shock. To see why, let us illustrate the evolution of the instantaneous welfare (in euros per year), defined as the function $F_{i}\left(G, g_{i}\right) e^{-\rho t}$ in equation (7), for the FB (in red), OL (in blue) and PO (in green) solutions in absence of any shock. Figure 2 displays this evolution for different time intervals and Table 3 provides the corresponding accumulative welfares (that is the area under the curve) of these zooms. We see in Table 3 that until the $5^{\text {th }}$ year of exploitation the most profitable solution is the FB solution, that gives up his position to the OL solution before the end of the $10^{\text {th }}$ year. It is not until the $30^{\text {th }}$ year onward that the PO solution becomes the most profitable. This gives interesting insights in how the inefficiency of private solutions is distributed

\footnotetext{
${ }^{5}$ Solutions of welfare in Tables 5 and 6 are associated to individual strategies.
} 
over time. ${ }^{6}$

\begin{tabular}{ccc} 
& {$\left[\mathbf{0}, \mathbf{t}_{\mathbf{a}}\right]$} & {$[\mathbf{0 ,} \boldsymbol{\infty}]$} \\
\hline PO & 111462 & 146658 \\
OL & 114886 & 132265 \\
FB & 101039 & 109180 \\
\hline PO-OL & -3424 & 14393 \\
OL-FB & 13847 & 23085 \\
PO-FB & 10423 & 37478 \\
\hline
\end{tabular}

Table 2: Welfare (and differences of welfare) obtained by the PO, OL and FB strategies (respectively between solutions) in thousands of Euros, when $r_{1}-r_{2}=70$ and for the date of occurrence $t_{a}=20$.

\begin{tabular}{ccccc} 
& {$[\mathbf{0}, \mathbf{5}]$} & {$[\mathbf{0}, \mathbf{1 0}]$} & {$[\mathbf{0}, \mathbf{2 0}]$} & {$[\mathbf{0 ,} \mathbf{3 0}]$} \\
\hline PO & 45997 & 75471 & 109655 & 127758 \\
OL & 66880 & 93282 & 114894 & 124670 \\
FB & 70257 & 89395 & 101100 & 105741 \\
\hline PO-OL & -20883 & -17811 & -5239 & 3088 \\
OL-FB & -3377 & 3887 & 13794 & 18929 \\
PO-FB & -24260 & -13924 & 8555 & 22017 \\
\hline
\end{tabular}

Table 3: Welfare (and differences of welfare) obtained by the PO, OL and FB strategies (resp. between solutions) in thousands of Euros, for the problem without shock $\left(r_{1}=r_{2}=\right.$ $360)$ in $[0, \mathrm{t}]$ for $\mathrm{t}=5,10,20$ and 30.

In what follows, we analyze how previous results change according to the intensity and date of occurrence of the shock.

\footnotetext{
${ }^{6}$ This does not mean that there is an optimal time in order to implement policy instruments. Policy measures should be implemented over the whole planning horizon, as shown in Proposition 4, in order to reach the PO path.
} 

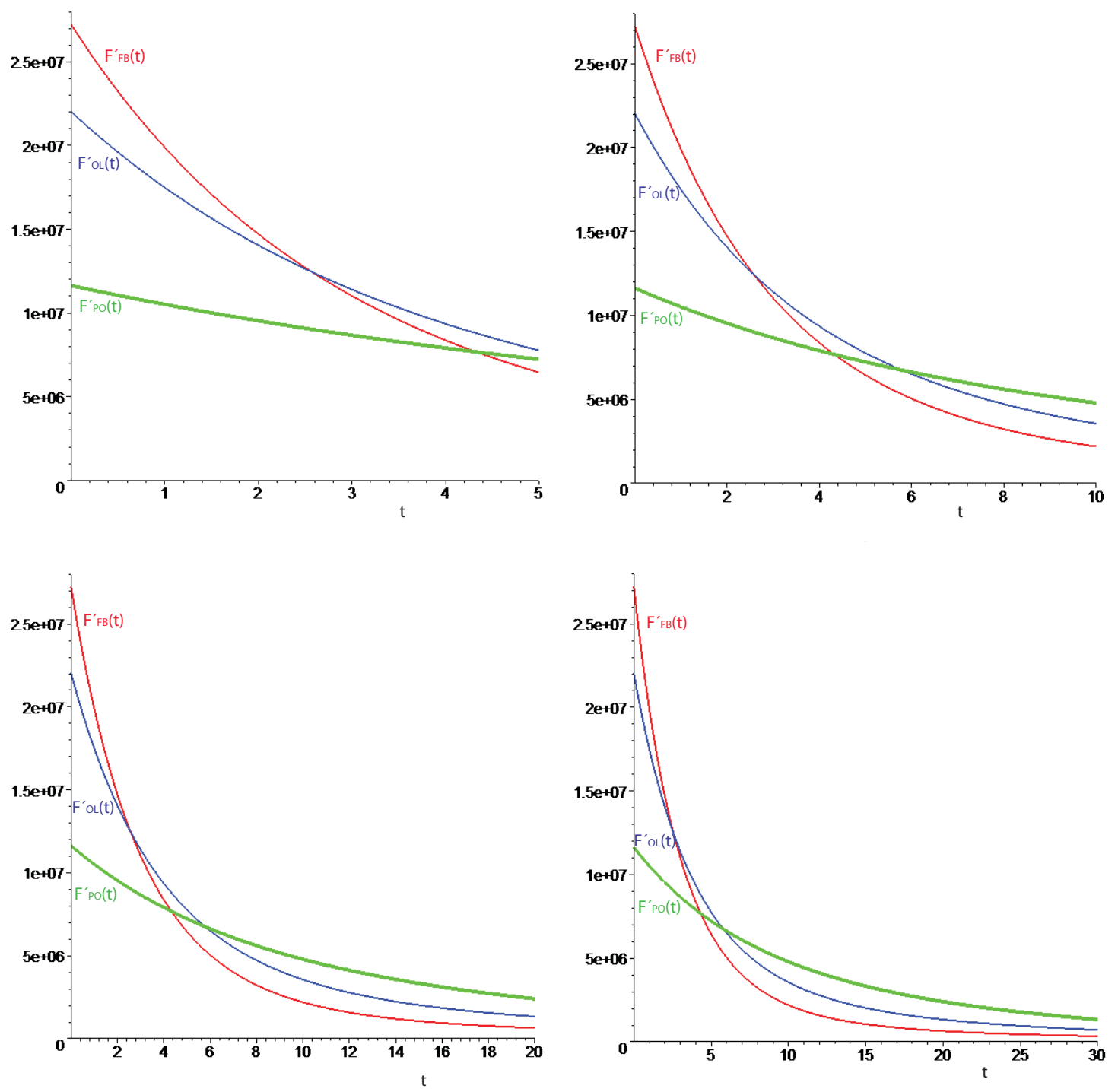

Figure 2: Evolution of $F_{i}^{\prime}\left(G, g_{i}\right)=F_{i}\left(G, g_{i}\right) e^{-\rho t}$ (in Euros per year) obtained by the PO (in green), OL (in blue) and FB (in red) strategies for the problem without shock $\left(r_{1}=r_{2}=360\right)$. Zoom in $[0,5]$ (left-top side), [0, 10] (right-top side), [0, 20] (left-bottom side) and $[0,30]$ (right-bottom side).

\subsubsection{Variation of the intensity of the shock}

In this section, we compare the efficiency, in terms of stocks and welfare, of the open-loop and feedback solutions for shocks of different intensities. For example, in Figure 3 we 

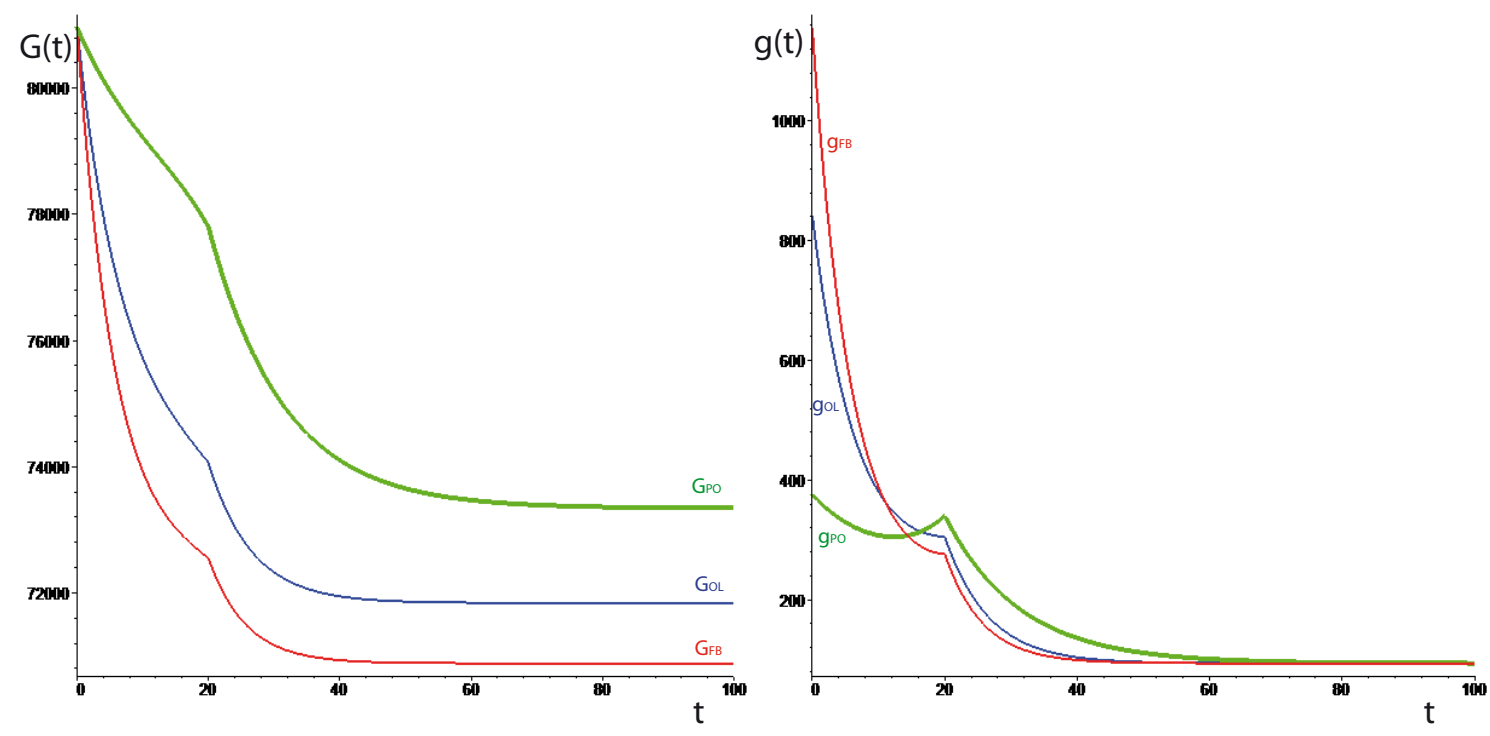

Figure 3: Solutions of $G^{*}(t)$ (left-hand side) in millions of cubic meters and $g^{*}(t)$ (righthand side) in millions of cubic meters per year : the pareto optimum (in green), the open-loop (in blue) and the feedback cases (in red), when $r_{1}-r_{2}=210$ and $t_{a}=20$ years.

simulate a shock of $210 \mathrm{Mm}^{3}$ /year, which is about $140 \mathrm{Mm}^{3}$ /year more intense than the shock described in the previous section (and illustrated in Figure 1), but takes place at the same date.

First of all, we analyze the problem at the steady state and quantify results presented in propositions 2 and 3 . We note that the cost and the strategic effects remain positive. We also note that cost and strategic effects decrease with the more intense shock. Hence, we numerically confirm one of our main theoretical result in Proposition 3: the greater the value of the shock, the smaller the cost and strategic effects, defined as the differences between steady state stock solutions, and then the smaller the inefficiency from private solutions in terms of stocks. Moreover, we can observe that cost and strategic effects are half as high as that of the mid-intense shock of $70 \mathrm{Mm}^{3}$ year, which is three times less intense. This means that cost and strategic effects do not vary proportionally with a change on the intensity of the shock. Thus we add to the previous result the finding that inefficiency decreases with more intense shocks but less than proportionally. We confirm 
this in Table 4 where other simulations for shocks of different intensities are illustrated.

\begin{tabular}{ccccc}
$r_{1}-r_{2}$ & 0 & 30 & 70 & 210 \\
\hline PO-OL & 3600 & 3300 & 2899 & 1500 \\
OL-FB & 2295 & 2104 & 1850 & 956 \\
PO-FB & 5895 & 5404 & 4749 & 2456 \\
\hline
\end{tabular}

Table 4: Differences between stock values in millions of $\mathrm{m}^{3}$ at the steady state.

\begin{tabular}{ccc|cc|cc|cc}
$r_{1}-r_{2}$ & \multicolumn{2}{c|}{0} & \multicolumn{2}{|c|}{30} & \multicolumn{2}{|c}{70} & \multicolumn{2}{c}{210} \\
\hline & {$\left[\mathbf{0}, \mathbf{t}_{\mathbf{a}}\right]$} & $\mathbf{[ 0 , \infty}]$ & {$\left[\mathbf{0}, \mathbf{t}_{\mathbf{a}}\right]$} & $\mathbf{[ 0 , \infty}]$ & {$\left[\mathbf{0}, \mathbf{t}_{\mathbf{a}}\right]$} & {$[\mathbf{0}, \boldsymbol{\infty}]$} & {$\left[\mathbf{0}, \mathbf{t}_{\mathbf{a}}\right]$} & {$[\mathbf{0}, \boldsymbol{\infty}]$} \\
\hline PO & 109655 & 153534 & 110446 & 150451 & 111462 & 146658 & 114666 & 136234 \\
OL & 114894 & 138564 & 114908 & 135750 & 114886 & 132265 & 114445 & 122475 \\
FB & 101100 & 112269 & 101074 & 110879 & 101039 & 109180 & 100900 & 104637 \\
\hline PO-OL & -5239 & 14970 & -4462 & 14701 & -3424 & 14393 & 221 & 13759 \\
OL-FB & 13794 & 26295 & 13834 & 24871 & 13847 & 23085 & 13545 & 17838 \\
PO-FB & 8555 & 41265 & 9372 & 39572 & 10423 & 37478 & 13766 & 31597 \\
\hline
\end{tabular}

Table 5: Welfare (and differences of welfare) obtained by the PO, OL and FB strategies (respectively between solutions) (in thousands of Euros) for different values of the shock $r_{1}-r_{2}$ and for the date of occurrence $t_{a}=20$.

Secondly, in Table 5 , we can observe that the total welfare in $[0, \infty]$ decreases the more intense the shock (columns 3, 5, 7 and 9 first three rows). This result is logical in the sense that after the occurrence of the shock, extractions decrease the higher the intensity of the shock due to the decrease on water availability.

However, we can also observe that the differences of total welfare between solutions in $[0, \infty]$ (columns 3, 5, 7 and 9 , last three rows) decreases the more intense the shock. In other words, the inefficiency in terms of welfare is maximal for the case without shock. Let us observe that the maximum of 41265 (column 3 last row) exceeds by 3787 thousands Euros the value of the baseline case. Hence, we numerically show that the greater the value of the shock, the smaller the differences between solutions and then the smaller the 
inefficiency, not only in terms of steady state stocks but also in terms of welfare. As we advanced in section 5.1, this result is explained by the fact that the less abundant the water to share in the future, the smaller the extend to which inefficiencies from non-cooperative users develop.

Now, we analyze extraction behavior before the occurrence of the shock (before $t_{a}$ ) for the various solutions illustrated in Figure 3 . In $\left[0, t_{a}\right]$, total extractions in the feedback case $\left(9757 \mathrm{Mm}^{3}\right)$ remain higher than in the OL $\left(8810 \mathrm{Mm}^{3}\right)$ and PO $\left(6474 \mathrm{Mm}^{3}\right)$ cases. Moreover, in the three cases, we observe a more intense extraction behavior (see the righthand side of Figure 3) in comparison with the shock of mid-intensity of $70 \mathrm{Mm}^{3}$ /year (that was illustrated in Figure 1). In particular, total extractions increase by 85,427 and $430^{7}$ $\mathrm{Mm}^{3}$ for the $\mathrm{FB}, \mathrm{OL}$ and PO cases respectively when the intensity of the shock increases by around $140 \mathrm{Mm}^{3}$ /year. We can confirm this result for different types of shocks by observing the left-hand side of Figure 4, that illustrates the total amount of extractions until $t_{a}$ with respect to the value of the shock, $r_{1}-r_{2}$. We note that the impatience effect increases the higher the shock but this increase is more important in the PO and OL cases than in the FB case. This could be interpreted as the fact that an increase in the "impatience effect" is less needed when considering the "strategic" externality, because extractions in the FB case are already very important in the beginning of the exercise. This is due to the fact that farmers have more information about the state of the result in the FB case and then they may react earlier to the shock. Finally, we can study the differences in welfare between solutions in $\left[0, t_{a}\right]$ (see Table 5 , columns $2,4,6$ and 8 , last three rows): welfare obtained from PO-OL and OL-FB strategies vary in a non-monotonic way with respect to the value of the shock, reaching sometimes negative values. Again, the pareto optimum is not the most profitable solution, if we analyze the problem in $\left[0, t_{a}\right]$. OL is performing better until the $20^{\text {th }}$ year of exploitation. We will see in next section that the ranking of solutions in $\left[0, t_{a}\right]$ is driven by a change on $t_{a}$.

\footnotetext{
${ }^{7}$ These numbers are obtained by deducting total extractions before $t_{a}$ for a shock of $210 \mathrm{Mm}^{3} /$ year and a shock of $70 \mathrm{Mm}^{3}$ /year, more specifically, by computing the differences 9 757-9 672, 8 810-8 383 and 6 474-6 044 for the feedback, open loop and respectively, pareto optimal solutions.
} 

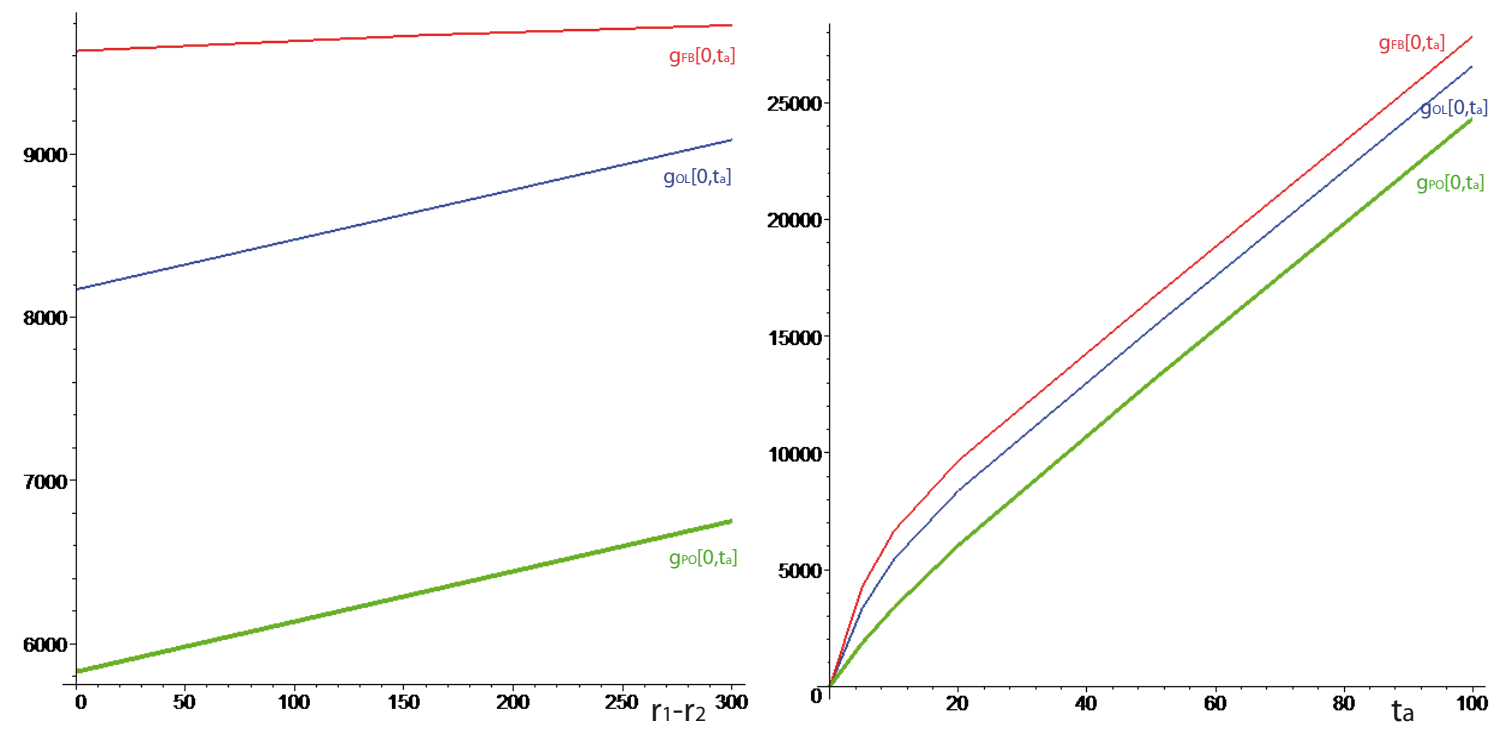

Figure 4: Total amount of extractions until $t_{a}$ in millions of $\mathrm{Mm}^{3}$ with respect to the value of the shock $r_{1}-r_{2}$ (left-hand side) and the date of occurrence $\left(t_{a}\right)$ (right-hand side), for the FB, OL and PO cases.

\subsubsection{Variation of the date of the shock}

After the analysis and estimation of extraction behavior in the different cases according to the intensity of the shock, we study the different solutions with respect to the date of occurrence of the shock. In Figure 5, we observe optimal solutions of stock $G^{*}(t)$ (on the left) and pumping rate $g^{*}(t)$ (on the right), in particular the pareto optimal (in green), the open loop (in blue) and the feedback solutions (in red), for a shock of mid-intensity of 70 millions of cubic meters per year $\left(\mathrm{Mm}^{3} /\right.$ year $)$, i.e. $r_{1}-r_{2}=70 \mathrm{Mm}^{3} /$ year occurring in the $5^{\text {th }}$ year of exploitation ( $t_{a}=5$ years). In what follows, we compare this shock with the previous shock illustrated in Figure 1, which has the same intensity but takes place 15 years later.

In the long-run, steady state stocks do not depend on the date of occurrence of the shock as we note in the analytical solutions (equations (15), (16) and (17)). Individual welfare does change with the date of occurrence of the shock, as shown in Table 6 . We note that total welfare in $[0, \infty]$ increases the later the shock occurs for all information structures 


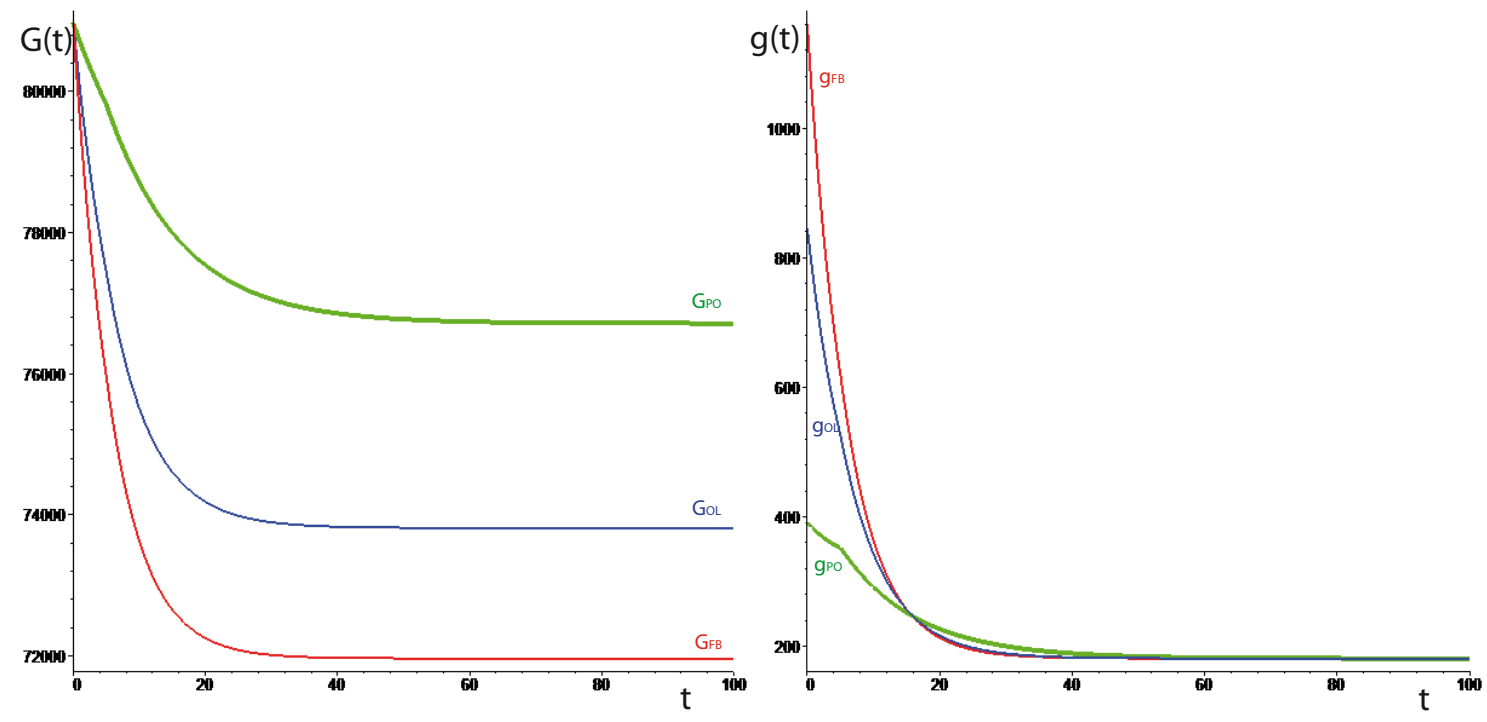

Figure 5: Solutions of $G^{*}(t)$ (left-hand side) in millions of cubic meters and $g^{*}(t)$ (righthand side) in millions of cubic meters per year : the pareto optimum (in green), the open-loop (in blue) and the feedback cases (in red), when $r_{1}-r_{2}=70$ and $t_{a}=5$ years.

(see columns 3, 5, 7 and 9 of the first three rows in Table 6). Logically, farmers are better off, if recharge rates are higher for longer times. The same result is obtained when we compute differences between solutions (see Table 6, columns 3, 5, 7 and 9, of the last three rows). As an example, the loss in total welfare derived from private exploitation (feedback solution) with respect to optimal exploitation, is greater of 3855 thousands of Euros when the shock occurs in $t_{a}=20$, instead of occurring earlier at $t_{a}=5$. Hence, the later the shock occurs, the greater the differences in welfare between solutions and therefore the greater the inefficiency. Farmers have more time to deploy their non-cooperative strategies and hence foster inefficiencies.

Next, if we do again an analysis of profitability in terms of stocks and welfare in $\left[0, t_{a}\right]$, we confirm that until the arrival of the shock, total extractions are higher in the FB case (4 $\left.254 \mathrm{Mm}^{3}\right)$ than in the OL $\left(3328 \mathrm{Mm}^{3}\right)$ and PO $\left(1845 \mathrm{Mm}^{3}\right)$ cases. Moreover, they are less important than in the previous shock that occurs at $t_{a}=20$. We note that this result is monotonic in time as shown in the right-hand side of Figure 4. In terms of welfare, we 


\begin{tabular}{c|cc|cc|cc|cc}
$t_{a}$ & \multicolumn{2}{|c|}{0} & \multicolumn{2}{|c|}{5} & \multicolumn{2}{|c}{20} & \multicolumn{2}{|c}{50} \\
& {$\left[\mathbf{0}, \mathbf{t}_{\mathbf{a}}\right]$} & {$[\mathbf{0}, \boldsymbol{\infty}]$} & {$\left[\mathbf{0}, \mathbf{t}_{\mathbf{a}}\right]$} & $\mathbf{[ 0 , \infty}]$ & {$\left[\mathbf{0}, \mathbf{t}_{\mathbf{a}}\right]$} & {$[\mathbf{0}, \boldsymbol{\infty}]$} & {$\left[\mathbf{0}, \mathbf{t}_{\mathbf{a}}\right]$} & {$[\mathbf{0}, \boldsymbol{\infty}]$} \\
\hline PO & 0 & 133375 & 48912 & 138348 & 111462 & 146658 & 144321 & 152021 \\
OL & 0 & 120991 & 68017 & 125350 & 114886 & 132265 & 133402 & 137086 \\
FB & 0 & 101058 & 70268 & 104725 & 101039 & 109180 & 109876 & 111594 \\
\hline PO-OL & 0 & 12384 & -19105 & 12998 & -3424 & 14393 & 11219 & 14935 \\
OL-FB & 0 & 19933 & -2251 & 20625 & 13847 & 23085 & 23526 & 25492 \\
PO-FB & 0 & 32317 & -21356 & 33623 & 10423 & 37478 & 34745 & 40427 \\
\hline
\end{tabular}

Table 6: Welfare (and differences of welfare) obtained by the PO, OL and FB strategies (respectively between solutions) (in thousands of Euros) for a shock of value $r_{1}-r_{2}=70$ $\mathrm{Mm}^{3}$ and different dates of occurrence.

can see in Table 6 (first three rows, columns 2, 4, 6 and 8) that welfare obtained in $\left[0, t_{a}\right]$ increases the later the shock occurs. This is linked to the fact that extractions are also more important when the shock takes place later for each information structure. However, if we analyze differences between solutions in $\left[0, t_{a}\right]$ (see last three rows of columns $2,4,6$ and 8 in Table 6), these are negative when the shock occurs at an earlier date $\left(t_{a}=5\right)$. Until the 5th year of exploitation, the feedback solution seems to be the most profitable strategy but it is not the optimal solution over the whole time horizon.

To briefly summarize this section, we have shown that in the long term (in $[0, \infty)$ ), inefficiency from private exploitation can be reduced when water availability decreases. If we analyze the problem until the $5^{\text {th }}$ year of exploitation of the aquifer, the feedback adaptation behaviors perform best, as they react earliest to the shock. Indeed, this is logical as feedback solutions correspond to the strategies where most information is available. If we analyze the problem until the $20^{\text {th }}$ year of exploitation, open-loop strategies are most profitable. Finally, over the whole time horizon, the pareto optimum is the most efficient solution. 


\section{Conclusions and discussion}

We have extended the analysis of a regime shift made in de Frutos Cachorro et al. (2014) [1] by taking into account different externalities which arise in the exploitation of a common groundwater resource, i.e. the dynamic and strategic interactions between users of the resource. Moreover, we have added to the existing literature which compares private and optimal exploitation of groundwater resources (Negri (1989) [8], Rubio and Casino (2001) [12]) the consideration of regime shifts following a decrease on the recharge rate of the aquifer.

We firstly show analytically that pumping cost and strategic effects decrease the greater the intensity of the shock. In order words, steady-state stock solutions get closer, and then inefficiency of private exploitation, defined as the difference between the pareto optimal and the feedback solutions, is reduced when the resource becomes more scarce. This result can seem counter-intuitive at first sight, but is in the same line with the Gisser and Sánchez effect (e.g. [4]). In their paper, Gisser and Sánchez prove that if the storage capacity (area of the aquifer times storativity) of the aquifer goes to infinity the difference between private and optimal solutions goes to zero. In their model, the recharge of the aquifer and the storage capacity define the evolution of the water table height, through an inverse relationship. In our context, instead of considering the problem of a big groundwater area we consider the problem of refilling the aquifer with small quantities of water. Although pumping cost and strategic effects decrease with greater shocks, there might still be need for policy intervention. We show analytically how the optimal quota should be imposed in order to correspond to the pareto optimal extraction path.

Next, we apply our game for the particular case of the Western la Mancha (WLM) aquifer. In the last decades, the WLM aquifer witnessed a critical decrease in water-table levels, due to the development of intensive irrigated agriculture, coupled with inefficient management regimes. Following the reasoning of Gisser and Sánchez in [4], a welfare analysis is necessary to justify an intervention in the management system.

We confirm that private solutions are inefficient compared to the pareto optimum, not only in terms of stocks but also in terms of welfare. In particular, in terms of stocks, the 
consideration of the strategic externality (feedback solution) exacerbates the overexploitation of the resource with respect to open loop strategies. These results are in agreement with the existing literature (Negri (1989) [8], Rubio and Casino (2001) [12]). Moreover, the inefficiency of private exploitation in terms of welfare decreases the higher the value of the shock and the earlier the shock occurs. This may be explained by the fact that there is less water to share in the future, and respectively, less time to compete for water and this is why the inefficiency cannot develop as easily as in a case with greater water availability. Nevertheless, the policy implication of our analysis is that a regulation through a centralized management of the Western la Mancha aquifer is still justified, because the pareto optimal solution allows positive efficiency gains, even though these gains are less important than in a case without shocks.

We also analyze how the inefficiency of private solutions is distributed over time. As in de Frutos Cachorro [1], we show that there is an "impatience effect", that is an increase in extractions before the occurrence of the shock. This "impatience effect" is more important in the FB case than in the PO and OL cases, increasing inefficiency in the most plausible non-cooperative solution. However, the FB solution is less influenced by an increase of the value of the shock than SO and OL solutions because farmers are already exploiting the resource stock more heavily in the beginning of the exercise than in the other solution cases. Indeed, farmers have extra information about the stock levels in the FB case, and then could adapt earlier to the shock. When the shock arrives at an earlier date, the FB (and then the OL solution) is the solution which entails the greatest gains in the short run, i.e. before the occurrence of the shock. This can explain why private farmers could be attracted by this solution. Nevertheless, the pareto optimum remains the most efficient solution over the entire time horizon. As we have mentioned previously, in order to bring the individual farmer to adopt the optimal solution path, licences could be imposed to bring the farmer from the FB solution path back to the PO solution path. Of course, those policies rely entirely on the individual farmer's faith to be compensated in future periods.

Some possible extensions of the article should also be mentioned. Firstly, we can introduce uncertainty in the model, for example on the date of the shock, as realized in [1], or on the intensity of the shock. Secondly, we could consider the date and the intensity of 
the shock as decision variables of the resource manager ${ }^{8}$. To tackle this issue it would be necessary to redefine the objective function taking into account the goal of water extraction. As the objective is defined currently, it is optimal to never implement the shock. With an objective that takes into account the trade-off between delivering water to farmers or to other users, the socially optimal date and intensity of the shock could be computed. Mathematically, such a problem could be solved in a Stackelberg game. We can also study as in Tsur and Zemel (2004) [14] an endogenous shock, that is an event whose occurrence is determined solely by the exploitation policy. Finally, it would be interesting to introduce heterogeneities between groups of farmers.

\footnotetext{
${ }^{8}$ We thank an anonymous referee for indicating this possibility.
} 


\section{A Resolution for the open-loop case}

We are going to solve the open-loop case proceeding firstly between $t_{a}$ and $\infty$. The Hamiltonian of this problem is:

$H_{i}=F_{i}\left(G, g_{i}\right)+\pi_{i}\left(r_{2}-(1-\alpha) \sum_{i=1}^{M} g_{i}\right)=\frac{a}{b} g_{i}-\frac{1}{2 b \theta_{i}} g_{i}^{2}-(z-c G) g_{i}+\pi_{i}\left(r_{2}-(1-\alpha) \sum_{i=1}^{M} g_{i}\right)$.

Applying the maximum principle and assuming interior solutions, we have the usual first order conditions:

$$
\begin{gathered}
\frac{\partial H_{i}}{\partial g_{i}}=0 \Rightarrow \frac{a}{b}-z+c G-\frac{1}{b \theta_{i}} g_{i}-\pi_{i}(1-\alpha)=0 \\
\dot{\pi}_{i}=-\frac{\partial H_{i}}{\partial G}+\rho \pi_{i} \Rightarrow \dot{\pi}_{i}=-c g_{i}+\rho \pi_{i} .
\end{gathered}
$$

The equilibrium of the open-loop game is obtained by solving $M$ strategies which verify the conditions $(22)$ and $(23)(\mathrm{i}=1 . . \mathrm{M})$, i.e. a linear system of $2 M$ equations. To simplify the analytical resolution of the problem, we assume that players are symmetric, $\theta_{i}=\frac{1}{M}$, $g=g_{i}$ and $\pi=\pi_{i}$. From (22), we find the optimal rate of extraction as a function of the resource stock and the shadow price:

$$
g=\frac{1}{M}(a-z b+c b G-\pi b(1-\alpha))
$$

Substituting (24) in the second part of equation (12) and adjoint variable (23), we have the following dynamic system:

$$
\begin{gathered}
\dot{G}=r_{2}-(1-\alpha)(a-z b)-c b(1-\alpha) G+\pi b(1-\alpha)^{2}, \\
\dot{\pi}=\frac{1}{M}\left(-c(a-z b)-c^{2} b G+c b(1-\alpha)+\rho M\right) \pi,
\end{gathered}
$$

which allows us to find the roots of the characteristic polynom: 


$$
\begin{gathered}
\beta_{1,2}=\frac{\rho M+c(1-\alpha) b(1-M)}{2 M} \\
\pm \frac{\sqrt{\rho^{2} M^{2}+c b(1-\alpha)\left(-2 M(1-\alpha) c b+c(1-\alpha) b\left(1+M^{2}\right)+2 \rho M(1+M)\right)}}{2 M} .
\end{gathered}
$$

From equations (24), (25) and (26), with $\dot{G}=0$ and $\dot{\pi}=0$, we find the steady state of the problem:

$$
\begin{gathered}
g_{\infty}^{O L}=\frac{r_{2}}{(1-\alpha) M}, \\
\pi_{\infty}^{O L}=\frac{c r_{2}}{M \rho(1-\alpha)}, \\
G_{\infty}^{O L}=\frac{r_{2}}{c b(1-\alpha)}+\frac{r_{2}}{M \rho}-\frac{a}{c b}+\frac{z}{c} .
\end{gathered}
$$

Assuming parameters are positives, $g_{\infty}$ and $\pi_{\infty}$ (equations (28) and (29)) have always positives values. Moreover, in what follows, we assume parameters such as the value of $G_{\infty}$ (equation (30)) is also positive.

Finally, we find optimal extraction path with $\beta_{2}$, the negative root:

$$
\begin{gathered}
G^{O L+}(t)=e^{\beta_{2}\left(t-t_{a}\right)}\left(G_{t_{a}}-G_{\infty}^{O L}\right)+G_{\infty}^{O L}, \\
g^{O L+}(t)=\frac{r_{2}}{(1-\alpha) M}-\frac{\beta_{2}}{(1-\alpha) M} e^{\beta_{2}\left(t-t_{a}\right)}\left(G_{t a}-G_{\infty}^{O L}\right), \\
\pi^{O L+}(t)=e^{\beta_{2}\left(t-t_{a}\right)}\left(\pi_{t a}-\pi_{\infty}^{O L}\right)+\pi_{\infty}^{O L},
\end{gathered}
$$

and,

$$
\pi_{t a}=\frac{a}{b(1-\alpha)}-\frac{z-c G_{t a}}{(1-\alpha)}-\frac{1}{b(1-\alpha)^{2}}\left(r_{2}-\beta_{2}\left(G_{t a}-G_{\infty}^{O L}\right)\right),
$$


which is obtained from equations (22) and (25).

In a second step, we will solve the problem between 0 and $t_{a}$. In this period, the Hamiltonian of the problem is described by:

$H_{i}=F_{i}\left(G, g_{i}\right)+\pi_{i}\left(r_{1}-(1-\alpha) \sum_{i=1}^{M} g_{i}\right)=\frac{a}{b} g_{i}-\frac{1}{2 b \theta_{i}} g_{i}^{2}-(z-c G) g_{i}+\pi_{i}\left(r_{1}-(1-\alpha) \sum_{i=1}^{M} g_{i}\right)$.

We use the same principle of resolution than previously. We have first order conditions (equations $(21),(22),(23)$ with $r_{2}=r_{1}$ ) by applying the maximum principle. Moreover, we assume that players are symmetric.

In a finite horizon problem, we write solutions as described below:

$$
\begin{aligned}
& G^{O L-}(t)=C_{1} e^{\beta_{1} t}+C_{2} e^{\beta_{2} t}+C_{3}, \\
& \pi^{O L-}(t)=D_{1} e^{\beta_{1} t}+D_{2} e^{\beta_{2} t}+D_{3} .
\end{aligned}
$$

Substituting $G^{O L-}(t)$ and $\pi^{O L-}(t)$ (equations (36) and (37)) in first order conditions (22), (23), and taking into account boundary conditions $G(0)=G_{0}$ and $\pi\left(t_{a}\right)=\pi^{O L+}\left(t_{a}\right)$, we obtain a system of 6 equations with 6 unknowns $\left(C_{i}, D_{i}\right.$ with $\left.\mathrm{i}=1,2,3\right)$. We find the following solutions to the system: ${ }^{9}$

$$
C_{1}=\frac{-(1-\alpha) b\left(-\rho M c r_{1}+\rho M c(1-\alpha)^{2} b \pi_{t a}-(1-\alpha) c^{2} b r_{1}\right)-c^{2} e^{\beta_{2} t_{a}} \rho M(1-\alpha) b G_{0}}{D_{1}}
$$

$\frac{+c e^{\beta_{2} t_{a}} \rho M\left(r_{1}+(1-\alpha) z b-(1-\alpha) a\right)+c r_{1} M \beta_{2}-(1-\alpha) \rho M^{2} \pi_{t a}\left(\beta_{2}-\rho\right)+c^{2} e^{\beta_{2} t_{a}} b(1-\alpha) r_{1}}{D_{1}}$

$$
\frac{\left.+c e^{\beta_{2} t_{a}} \rho M\left(r_{1}+(1-\alpha) z b-(1-\alpha) a\right)+c r_{1} M \beta_{2}-(1-\alpha) \rho M^{2} \pi_{t a}\left(\beta_{2}-\rho\right)\right)}{D_{1}},
$$

\footnotetext{
${ }^{9}$ Solution of $D_{3}$ is not detailed here, but they are available from authors request.
} 
with

$D_{1}=\rho M\left(-e^{\beta_{1} t_{a}} \beta_{1}\left(\rho M+c b(1-\alpha)-M \beta_{2}\right)+(1-\alpha) c b\left(-(1-\alpha) c b\left(e^{\beta_{2} t_{a}}-e^{\beta_{1} t_{a}}\right)+M e^{\beta_{1} t_{a}}\left(\beta_{2}-\rho\right)\right)\right)$,

$C_{2}=\frac{-\left(-\rho M-c b(1-\alpha)+\beta_{2} M\right)\left(-c^{2} b^{2}(1-\alpha)^{2} r_{1}-\rho M e^{\beta_{1} t_{a}} c b(1-\alpha) G_{0}\left(\beta_{1}+c b(1-\alpha)\right)\right.}{D_{2}}$

$\frac{+c b^{2}(1-\alpha)^{3} \rho M \pi_{t a}+e^{\beta_{1} t_{a}}\left(\beta_{1}+c b(1-\alpha)\right)\left(\rho M\left(r_{1}-(a-z b)(1-\alpha)\right)+c b(1-\alpha) r_{1}\right)}{D_{2}}$,

with

$$
\begin{gathered}
D_{2}=c b(1-\alpha) \rho M\left(\left(\beta_{2}-\rho M-c b(1-\alpha)+M \beta_{2}\right)\right), \\
+c b(1-\alpha)\left(e^{\beta_{2} t_{a}} c b(1-\alpha)+e^{\beta_{1} t_{a}}\left(-c b(1-\alpha)\left(\beta_{2}-\rho\right) M\right)\right), \\
C_{3}=\frac{\rho M\left(r_{1}-(a-z b)(1-\alpha)\right)+c b(1-\alpha) r_{1}}{c b(1-\alpha) \rho M},
\end{gathered}
$$

and $\pi_{t a}$ described in equation (34). Finally, taking into account that $G(t)$ is a continuous function $\left(G^{O L-}\left(t_{a}\right)=G^{O L+}\left(t_{a}\right)\right)$, we find optimal solutions for the open loop game, that is $G^{O L}(t), g^{O L}(t)$ and $\pi^{O L}(t)$.

\section{B Resolution of the feedback case}

Now, for the feedback case, we solve as previously first the problem between $t_{a}$ and $\infty$.

The problem of player $\mathrm{i}$ is: 


$$
\max _{g_{i}(.)} \int_{t_{a}}^{\infty} \quad F_{i}\left(G, g_{i}\right) e^{-\rho\left(t-t_{a}\right)} d t
$$

with $F_{i}\left(G, g_{i}\right)$ (equation (8)), constrained by the dynamics

$$
\dot{G}=-(1-\alpha)\left(g_{i}+\sum_{\substack{j=1, j \neq i}}^{M} a_{j} G+b_{j}\right)+r
$$

with $r=r_{2}$ and conditions (11) and $G\left(t_{a}\right)=G_{t a}$.

For each player $i(i=1 . . M)$ the optimal value of the resource, $V^{i}(G)$, have to verify the Hamilton-Jacobi-Bellman equation:

$$
\rho V^{i}(G)=\max _{g_{i}}\left(F_{i}\left(G, g_{i}\right)-V_{G}^{i}(G)\left(r_{2}-(1-\alpha)\left(g_{i}+\sum_{\substack{j=1, j \neq i}}^{M} g_{j}\right)\right), \quad i=1 . . M\right.
$$

with

$$
\begin{gathered}
V^{i}(G)=A G^{2}+B G+C, \\
g_{j}=a_{j} G+b_{j} .
\end{gathered}
$$

Assuming that players are symmetric, we rewrite the equation (43) as follows:

$$
\rho V^{i}(G)=\max _{g_{i}}\left(F_{i}\left(G, g_{i}\right)-V_{G}^{i}(G)\left(r_{2}-(1-\alpha)\left(g_{i}+(M-1) g_{j}\right)\right)\right), \quad i=1 . . M,
$$

Next, solving the problem on the right hand-side of (46), we find the optimal pumping rate of player i, $g_{i}^{*}$ :

$$
g_{i}^{*}=a_{i}^{*} G+b_{i}^{*}
$$

with,

$$
a_{i}^{*}=\frac{b(c-(1-\alpha) 2 A)}{M},
$$




$$
b_{i}^{*}=\frac{a-z b-b(1-\alpha) B}{M},
$$

and $\mathrm{A}$ and $\mathrm{B}$ in equation (85), appendix D.

Substituting now $g_{i}^{*}$ on the right hand-side of (46) and equalizing the left and hand sides of the equation, we obtain optimal values of coefficients $A, B$ and $C$ of $V^{i}$ with regards to variables $a_{j}$ and $b_{j}$, the coefficients of the pumping rate function of player $j$ $(j \neq i)$. Moreover, replacing $A^{*}$ and $B^{*}$ (see formulas in (85)) in equations (48) and (49), and taking into account that players are symmetric, that is, $g_{1}(t)=g_{i}(t)$ for any $t=t_{a} . . \infty$ (and then $a_{1}=a_{i}$ and $b_{1}=b_{i}$ for any $i,(i=1 . . M)$, we find optimal values of coefficients of the pumping rate function of the individual player, $a_{1}^{*}$ and $b_{1}^{*}$.

Finally, substituting $a_{1}^{*}$ and $b_{1}^{*}$ in $(47)$ and replacing $g_{1}^{*}=g_{i}^{*}$ for any $i,(i=1 . . M)$ in the dynamics of the aquifer (42) with $r=r_{2}$, we may solve the differential equation (42), constrained by condition $G\left(t_{a}\right)=G_{t a}$. Thus, we obtain optimal solutions of the feedback problem, $G^{F B+}(t), g^{F B+}(t)$ and the optimal value function $V^{*}(G)$ in $\left[t_{a}, \infty\right)$ :

$$
\begin{gathered}
G^{F B}(t)=e^{-M(1-\alpha) a_{1}^{*}\left(t-t_{a}\right)}\left(G_{t a}-G_{\infty}\right)+G_{\infty}, \\
g^{F B}(t)=\frac{r_{2}}{M(1-\alpha)}+a_{1}^{*} e^{-M(1-\alpha) a_{1}^{*}\left(t-t_{a}\right)}\left(G_{t a}-G_{\infty}\right),
\end{gathered}
$$

and

$$
V^{+}(G)=A^{*} G^{2}+B^{*} G+C^{*}
$$

with,

$$
G_{\infty}^{F B}=\frac{r_{2}}{M(1-\alpha) a_{1}^{*}}-\frac{b_{1}^{*}}{a_{1}^{*}}
$$

and $b_{1}^{*}, a_{1}^{*}$ described in equations (48) and (49). 
Next, we solve the problem between 0 and $t_{a}$. The value function of the problem of player i, $V^{i}(t, G){ }^{10}$ verifies the Hamilton-Jacobi-Bellman equation:

$$
\rho V^{i}(t, G)-V_{t}^{i}(t, G)=\max _{g_{i}}\left(F_{i}\left(G, g_{i}\right)-V_{G}^{i}(G)\left(r_{1}-(1-\alpha)\left(g_{i}+\sum_{\substack{j=1, j \neq i}}^{M} g_{j}\right)\right)\right), \quad i=1 . . M
$$

with

$$
\begin{gathered}
V^{i}(t, G)=A(t) G^{2}+B(t) G+C(t), \\
g_{j}(t)=a_{j}(t) G+b_{j}(t),
\end{gathered}
$$

and the transversality condition,

$$
V^{i}\left(t_{a}, G_{t a}\right)=V^{+}\left(G_{t a}\right) .
$$

The value of $V^{+}\left(G_{t a}\right)$ is obtained from the first step of resolution of the problem, and is described in equation (51). To solve the second step of the problem, we are going to use a similar process as previously. The challenge here lies in the fact that strategies of players depend on the stock of the resource $G$ and on functions $a_{j}(t)$ and $b_{j}(t)$ in an independent way. So, the resolution of the problem is largely numerical.

Once again, assuming players are symmetric, we rewrite (53) as

$$
\rho V^{i}(t, G)-V_{t}^{i}(t, G)=\max _{g_{i}}\left(F_{i}\left(G, g_{i}\right)-V_{G}^{i}(G)\left(r_{1}-(1-\alpha)\left(g_{i}+(M-1) g_{j}\right)\right), \quad i=1 . . M,\right.
$$

and we solve the right part of equation (57). We then find the expression (47) that is the optimal pumping rate of player i, $g_{i}^{*}(t)$, with $a_{i}=a_{i}(t)$ and $b_{i}=b_{i}(t)$, described in equations (48), (49) and $A=A(t), B=B(t)$, which are now functions that depend on $\mathrm{t}$.

Now, substituting $g_{i}^{*}(t)$ in the right part of equation (53), and equalizing the right and left parts of the equation, we have to solve a system of 3 differential equations in $A(t), B(t)$

\footnotetext{
${ }^{10}$ We remind that in this type of problem with a finite horizon planning, the value function has to be described as a function that depends on $\mathrm{G}$ and $\mathrm{t}$ independently.
} 
and $C(t)$, which are coefficients of the value function $V(t, G)$, between $t=0$ and $t=t_{a}$, (see equation (54)), taking into account boundary conditions:

$$
A\left(t_{a}\right)=A^{*}, B\left(t_{a}\right)=B^{*}, C\left(t_{a}\right)=C^{*},
$$

derived from the transversality condition (56):

$$
V^{-}\left(G_{t a}, t_{a}\right)=A\left(t_{a}\right) G_{t_{a}}^{2}+B\left(t_{a}\right) G_{t_{a}}+C\left(t_{a}\right)=V^{+}\left(G_{t a}\right)
$$

At this stage, we obtain $A^{*}(t)$ and $B^{*}(t)$ by a numerical approximation method. Substituting $A^{*}(t)$ and $B^{*}(t)$ in expression $g_{i}^{*}(t)$, we find the optimal values of coefficients $b^{*}(t)$ and $a^{*}(t)$ of the pumping rate. Next, we substitute these values in the equation of motion (42) with $r=r_{1}$. Finally, we obtain the numerical solution of the feedback problem between 0 and $t_{a}$, that is $G^{F B-}(t)$ and $g^{F B-}(t)$, where the initial condition $G(0)=G_{0}$, is given.

\section{Resolution of the pareto optimum}

To solve this problem, we separate it into two parts and proceed by backward induction. First, we solve the maximization between $t_{a}$ and $\infty$. The problem of the social planner is to find $\phi\left(G_{t a}\right)$,

$$
\phi\left(G_{t a}\right)=\max _{g_{i}(.)} \int_{t_{a}}^{\infty} \quad \sum_{i=1}^{M} F_{i}\left(G, g_{i}\right) e^{-\rho\left(t-t_{a}\right)} d t,
$$

with $F_{i}\left(G, g_{i}\right)$ (equation (8)), constrained by equation (9) with $r=r_{2}$ and conditions (11) and $G\left(t_{a}\right)=G_{t a}$.

The Hamiltonian of this problem is given by:

$$
H=\sum_{i=1}^{M}\left(\frac{a}{b} g_{i}-\frac{1}{2 b \theta_{i}} g_{i}^{2}-(z-c G) g_{i}\right)+\lambda\left(-(1-\alpha) \sum_{i=1}^{M} g_{i}+r_{2}\right),
$$

where $\lambda$ is the adjoint variable. Applying the maximum principle and assuming interior solutions, we have the usual first order conditions: 


$$
\begin{gathered}
\frac{\partial H}{\partial g_{i}}=0 \Rightarrow \frac{a}{b}-\frac{1}{b \theta_{i}} g_{i}-(z-c G)-\lambda(1-\alpha)=0, \quad i=1 . . M \\
\dot{\lambda}=-\frac{\partial H}{\partial G}+\rho \lambda \Rightarrow \dot{\lambda}=-c \sum_{i=1}^{M} g_{i}+\rho \lambda, \quad i=1 . . M .
\end{gathered}
$$

We assume that players are symmetric in order to simplify the analytical resolution of the problem. Thus, $\theta_{i}=\frac{1}{M}$ and $g=g_{i}$.

From (59), we find the optimal extraction volume as a function of the resource stock and the shadow price:

$$
g=\frac{1}{M}(a-z b+c b G-\lambda b(1-\alpha)) .
$$

Substituting (61) in the second part of equation of motion (12) and equation of adjoint variable (60), we have the following dynamic system:

$$
\begin{gathered}
\dot{G}=r_{2}-(1-\alpha)(a-z b)-c b(1-\alpha) G+\lambda b(1-\alpha)^{2}, \\
\dot{\lambda}=-c(a-z b)-c^{2} b G+(c b(1-\alpha)+\rho) \lambda,
\end{gathered}
$$

which allows us to find the roots of the characteristic polynom:

$$
\rho_{1,2}=\frac{\rho \pm \sqrt{\rho^{2}+4 c b(1-\alpha) \rho}}{2} .
$$

From equations (61), (62) and (63), with $\dot{G}=0$ and $\dot{\lambda}=0$, we find the steady state of the pareto optimum problem:

$$
\begin{gathered}
g_{\infty}^{P O}=\frac{r_{2}}{(1-\alpha) M}, \\
\lambda_{\infty}^{P O}=\frac{c r_{2}}{\rho(1-\alpha)}, \\
G_{\infty}^{P O}=\frac{r_{2}}{c b(1-\alpha)}+\frac{r_{2}}{\rho}-\frac{a}{c b}+\frac{z}{c} .
\end{gathered}
$$


Since we assume that all parameters are positive, $g_{\infty}$ and $\lambda_{\infty}$ (equations (65) and (66)) are always positive. Moreover, in what follows, we consider parameters such that $G_{\infty}$ (equation (67)) is positive.

Finally, we have the optimal extraction paths from $t=t_{a}$, with $\rho_{2}$, the negative root:

$$
\begin{gathered}
G^{P O+}(t)=e^{\rho_{2}\left(t-t_{a}\right)}\left(G_{t a}-G_{\infty}\right)+G_{\infty}, \\
\lambda^{P O+}(t)=e^{\rho_{2}\left(t-t_{a}\right)}\left(\lambda_{t a}-\lambda_{\infty}\right)+\lambda_{\infty}, \\
g^{P O+}(t)=\frac{r_{2}}{(1-\alpha) M}-\frac{\rho_{2}}{(1-\alpha) M}\left(G_{t a}-G_{\infty}\right) e^{\rho_{2}\left(t-t_{a}\right)},
\end{gathered}
$$

with,

$$
\lambda_{t a}=\frac{a}{b(1-\alpha)}+\frac{-z+c G_{t a}}{(1-\alpha)}-\frac{r_{2}}{b(1-\alpha)^{2}}+\frac{1}{b(1-\alpha)^{2}} \rho_{2}\left(G_{t a}-G_{\infty}\right), \quad G_{t a} \quad \text { unknown. }
$$

Substituting (68) and (70) in problem (58), we can compute the scrap value, $\phi\left(G_{t a}\right)^{11}$ :

$$
\begin{gathered}
\phi\left(G_{t a}\right)=\epsilon+\kappa G_{t a}+\iota G_{t a}^{2}, \quad \text { with }^{12} \\
\kappa=\frac{-r_{2} \rho(4 c b(1-\alpha)+\rho)+\rho^{2}(1-\alpha)(a-z b)+4 c b(1-\alpha)^{2} \rho(a-z b)}{\rho(1-\alpha)^{2} b(\eta+\rho+4 c b(1-\alpha))} \\
+\frac{\left.(2 c b(1-\alpha)+\rho) r_{2} \eta-\rho(1-\alpha) \eta(a-z b)\right)}{\rho(1-\alpha)^{2} b(\eta+\rho+4 c b(1-\alpha))}, \\
\iota=-\frac{c(-4 c b(1-\alpha)-\rho+\eta)}{(1-\alpha)(\eta+\rho+4 c b(1-\alpha))}, \quad \text { and } \\
\eta=\sqrt{\rho} \sqrt{\rho+4 b c(1-\alpha)} .
\end{gathered}
$$

\footnotetext{
${ }^{11}$ We find that the expression $\phi\left(t a, G_{t a}\right)$ does not have the independent term $t_{a}$. In what follows, we write the scrap value function, $\phi\left(G_{t a}\right)$.

${ }^{12}$ We do not detail expression of $\epsilon$ because it is not necessary for the resolution of the problem, but it is available from the authors upon request
} 
We now turn to the second part of the problem, between 0 and $t_{a}$, considering the optimal solution for the first part. The problem for the social planner is now:

$$
\begin{gathered}
\max _{g_{i}(.)} \int_{0}^{t_{a}} \quad F_{i}\left(G, g_{i}\right) \quad e^{-\rho t} d t+e^{-\rho t_{a}} \phi\left(t_{a}, G_{t a}\right) \\
\dot{G}=-(1-\alpha) \sum_{i=1}^{M} g_{i}+r_{1} \\
G(0)=G_{0} \text { given, }
\end{gathered}
$$

and the transversality condition:

$$
\lambda\left(t_{a}\right)=\frac{\partial \phi\left(t a, G_{t a}\right)}{\partial G_{t a}}=2 \iota G_{t a}+\kappa,
$$

with $\phi\left(t a, G_{t a}\right)$ described by equation (71). The Hamiltonian can be written as:

$$
H=\sum_{i=1}^{M}\left(\frac{a}{b} g_{i}-\frac{1}{2 b \theta_{i}} g_{i}^{2}-(z-c G) g_{i}\right)+\lambda\left(-(1-\alpha) \sum_{i=1}^{M} g_{i}+r\right),
$$

where $\lambda$ is the adjoint variable. Applying the maximum principle and assuming interior solutions, we have the usual first order conditions (59) and (60). From this and equation of the motion of the state (76), we have the system of differential equations:

$$
\begin{gathered}
\dot{G}=r_{1}-(1-\alpha)(a-z b)-c b(1-\alpha) G+\lambda b(1-\alpha)^{2}, \\
\dot{\lambda}=-c(a-z b)-c^{2} b G+(c b(1-\alpha)+\rho) \lambda .
\end{gathered}
$$

We know that the solutions of the finite problem are now, of the shape:

$$
\begin{aligned}
& G^{P O-}(t)=A_{1} e^{\rho_{1} t}+A_{2} e^{\rho_{2} t}+A_{3}, \\
& \lambda^{P O-}(t)=B_{1} e^{\rho_{1} t}+B_{2} e^{\rho_{2} t}+B_{3},
\end{aligned}
$$

with,

$$
G^{P O-}(0)=A_{1}+A_{2}+A_{3}=G_{0},
$$




$$
\lambda\left(t_{a}\right)=B_{1} e^{\rho_{1} t a}+B_{2} e^{\rho_{2} t a}+B_{3}=2 \iota G_{t a}+\kappa,
$$

and $\rho_{1}, \rho_{2}$ described in equation (64). This constitutes a system of 6 equations and 6 unknowns, which we can solve to find optimal solutions for the problem for the first period, between 0 and $t_{a}$. We find optimal values of $A_{i}, B_{i}(i=1 . .3)^{13}$ :

$$
A_{i}=C_{1}^{i}+C_{2}^{i}\left(2 v G_{r}^{*}\left(t_{a}\right)+\tau\right), \quad \mathrm{i}=1,2,
$$

with,

$$
\begin{gathered}
C_{1}^{1}=\frac{b(1-\alpha)\left(\rho c\left(r_{1}+(1-\alpha) e^{\rho_{2} t_{a}}(a-z b)-r_{1} e^{\rho_{2} t_{a}}\right)-\rho_{2} c r_{1}\right)}{D^{1}} \\
+\frac{b(1-\alpha)\left((1-\alpha) c^{2}\left(r_{1} b+e^{\rho_{2} t_{a}} \rho b G_{0}-r_{1} b e^{\rho_{2} t_{a}}\right)\right)}{D^{1}}, \\
C_{2}^{1}=\frac{b(1-\alpha)^{2} \rho\left(\rho_{2}-\rho-c b(1-\alpha)\right)}{D^{1}}, \\
D^{1}=\rho\left((\rho 2-\rho) e^{\rho_{1} t_{a}} c b(1-\alpha)+c^{2} b^{2}(1-\alpha)^{2}\left(e^{\rho_{2} t_{a}}-e^{\rho_{1} t_{a}}\right)-c b(1-\alpha), \rho_{1} e^{\rho_{1} t_{a}}+\rho_{1} e^{\rho_{1} t_{a}}\left(\rho_{2}-\rho\right)\right), \\
C_{1}^{2}=\frac{-\left(c b(1-\alpha)+\rho-\rho_{2}\right)\left(\rho_{1} e^{\rho_{1} t_{a}} \rho\left((1-\alpha)(a-z b)-r_{1}\right)\right.}{D^{2}} \\
+\frac{c^{2} b^{2}(1-\alpha)^{2}\left(G_{0} \rho+r_{1}-r_{1} e^{\rho_{1} t_{a}}\right)}{D^{2}}, \\
C_{2}^{2}=\frac{\left(c b(1-\alpha)+\rho-\rho_{2}\right)\left(c b^{2}(1-\alpha)^{3} \rho\right)}{D^{2}}, \\
\frac{\left.+c b(1-\alpha)\left(\left(G_{0} \rho-r_{1}\right) e^{\rho_{1} t_{a}} \rho_{1}-\rho r_{1} e^{\rho_{1} t_{a}}\right)+c b(1-\alpha)^{2} e^{\rho_{1} t_{a}} \rho(a-z b)\right)}{D^{2}},
\end{gathered}
$$

\footnotetext{
${ }^{13}$ We do not provide detailed solutions of $B_{i}(i=1 . .3)$ because the equations are too long and they are not necessary for the proofs, however, they are available from the authors upon request.
} 


$$
\begin{aligned}
D^{2}= & c b(1-\alpha) \rho\left(\left(\rho_{2}-\rho\right) c b(1-\alpha) e^{\rho_{1} t_{a}}+c^{2} b^{2}(1-\alpha)^{2}\left(e^{\rho_{2} t_{a}}-e^{\rho_{1} t_{a}}\right)\right) \\
& +c b(1-\alpha) \rho\left(-c b(1-\alpha) e^{\rho_{1} t_{a}} \rho_{1}-\rho e^{\rho_{1} t_{a}} \rho_{1}+e^{\rho_{1} t_{a}} \rho_{1} \rho_{2}\right)
\end{aligned}
$$

and,

$$
A_{3}=-\frac{r_{1} \rho-\rho a+\rho z b+\rho \alpha a-\rho \alpha z b+c r_{1} b-c r_{1} b \alpha}{b \rho(\alpha-1) c} .
$$

Finally, considering the continuity of the function of the variable state, i.e. $G^{P O-}\left(t_{a}\right)=$ $G^{P O+}\left(t_{a}\right)$, we obtain optimal solution of the stock $G^{P O}(t)$ and water pumping $g^{P O}(t)$ for the pareto optimum problem.

\section{Steady states and proofs of propositions}

\section{D.1 Conditions for positive steady-state values.}

We have obtained:

$$
G_{\infty}^{F B}=\frac{r_{2}}{M(1-\alpha) a_{1}}-\frac{b_{1}}{a_{1}}
$$

with

$$
\begin{gathered}
a_{1}=\frac{b(c-(1-\alpha) 2 A)}{M}, \quad b_{1}=\frac{a-z b-b(1-\alpha) B}{M}, \\
A=\frac{M[2 c(1-\alpha)-\rho / b]+\sqrt{W}}{4(2 M-1)(1-\alpha)^{2}}, \quad B=\frac{-2 A\left[M r_{2}-M(a-b z)(1-\alpha)\right]-c(a-b z)}{(2 M-1)(1-\alpha)^{2} b 2 A-M \rho-M c b(1-\alpha)},
\end{gathered}
$$

and

$$
W=\frac{\rho^{2} M^{2}}{b^{2}}+\frac{4 M^{2} \rho(1-\alpha) c}{b}+4(M-1)^{2}(1-\alpha)^{2} c^{2}>0 .
$$

Stability condition implies that $2 A<\frac{c}{1-\alpha}$ and then $a_{1}>0$. To obtain $G_{\infty}^{F B}>0$ from (84) for all $r_{2}$ we must impose $b_{1}<0$.

Remind also that

$$
G_{\infty}^{P O}=\frac{r_{2}}{c b(1-\alpha)}+\frac{r_{2}}{\rho}-\frac{a}{c b}+\frac{z}{c}, \quad G_{\infty}^{O L}=\frac{r_{2}}{c b(1-\alpha)}+\frac{r_{2}}{M \rho}-\frac{a}{c b}+\frac{z}{c} .
$$


To have $G_{\infty}^{P O}>0$ and $G_{\infty}^{O L}>0$ for all $r_{2}$ we must impose $-a+b z>0$.

As we assume that all the parameters are positive, it is easy to verify that

$$
\lim _{r_{2} \rightarrow 0} G_{\infty}^{F B}=\lim _{r_{2} \rightarrow 0} G_{\infty}^{O L}=\lim _{r_{2} \rightarrow 0} G_{\infty}^{P O}=\frac{-a+z b}{c b}>0 .
$$

\section{D.2 Proof of proposition 1:}

Sign of $\frac{\partial G_{\infty}^{P O}}{\partial r_{2}}, \frac{\partial G_{\infty}^{O L}}{\partial r_{2}}$ and $\frac{\partial G_{\infty}^{F B}}{\partial r_{2}}$.

This is immediate from equations (15) and (16) for the pareto optimal and the openloop cases. Assuming $-a+b z>0$, it is enough to prove that the derivatives of expressions described in these equations with regard to $r_{2}$ are bigger than 0 .

In the feedback case, replacing expressions of equation (85) in (84) and differentiating with respect to $r_{2}$, it is clear that

$$
\frac{\partial G_{\infty}^{F B}}{\partial r_{2}}=\frac{1}{b(1-\alpha)[c-2 A(1-\alpha)]}>0
$$

due to the stability condition.

\section{D.3 Proof of proposition 2:}

Sign of $G_{\infty}^{F B}-G_{\infty}^{O L}$ and $G_{\infty}^{O L}-G_{\infty}^{P O}$.

Firstly, from equation (19), It is evident that

$$
G_{\infty}^{O L}-G_{\infty}^{P O}<0
$$

Next, replacing expressions of equation (85) in (20), we can see that

$$
G_{\infty}^{F B}-G_{\infty}^{O L}=-\frac{2 c b r_{2}(1-\alpha)(M-1)}{[M \rho+\sqrt{W} b] \rho M}<0
$$




\section{D.4 Proof of proposition 3:}

Sign of $\frac{\partial\left(G_{\infty}^{F B}-G_{\infty}^{O L}\right)}{\partial r_{2}}$ and $\frac{\partial\left(G_{\infty}^{O L}-G_{\infty}^{P O}\right)}{\partial r_{2}}$.

Finally, if we differentiate equations (19) and (86) with respect to $r_{2}$, it is immediate that

$$
\frac{\partial\left(G_{\infty}^{O L}-G_{\infty}^{P O}\right)}{\partial r_{2}}<0
$$

and

$$
\frac{\partial\left(G_{\infty}^{F B}-G_{\infty}^{O L}\right)}{\partial r_{2}}=-\frac{2 c b(1-\alpha)(M-1)}{[M \rho+\sqrt{W} b] \rho M}<0 .
$$

\section{D.5 Proof of proposition 4:}

We have to prove that $g=g^{P O}$ is a solution of the feedback problem constraint by $g \leq$ $\bar{g}(G)=g^{P O}$.

We solve as previously first the problem between $t_{a}$ and $\infty$. Considering the symmetric case, $V^{P O+}(G)$ and $g^{P O+}$ are the solutions of the Hamilton-Jacobi-Bellman equation:

$$
\rho V^{P O+}(G)=\max _{g}\left[M F(g, G)+V_{G}^{P O+}(G)(r-(1-\alpha) M g)\right]
$$

with

$$
\begin{gathered}
\frac{\partial F\left(g^{P O+}, G\right)}{\partial g}-V_{G}^{P O+}(G)(1-\alpha)=0, \forall G, \\
\rho V^{P O+}(G)=M F\left(g^{P O+}, G\right)+V_{G}^{P O+}(G)\left(r-(1-\alpha) M g^{P O+}\right), \forall G,
\end{gathered}
$$

and $r=r_{2}$.

Likewise, $V^{F B+}(G)$ and $g^{F B+}(G)$, constraint by a quota $\bar{g}(G)$ are the solution of the Hamilton-Jacobi-Bellman equation of player i:

$$
\rho V^{F B+}(G)=\max _{g \leq \bar{g}}\left[F(g, G)+V_{G}^{F B+}(G)\left(r-(1-\alpha)\left(g+\sum_{j \neq i} g_{j}(G)\right)\right)\right]
$$

with

$$
\begin{gathered}
\frac{\partial F\left(g^{F B+}, G\right)}{\partial g}-V_{G}^{F B+}(G)(1-\alpha)-\mu=0, \forall G, \quad \mu \geq 0, \\
\rho V^{F B+}(G)=F\left(g^{F B+}, G\right)+V_{G}^{F B+}(G)\left(r-(1-\alpha) M g^{F B+}\right), \forall G,
\end{gathered}
$$


and $r=r_{2}$.

The objective is to find $\bar{g}(G)$ such that $g^{F B+}=g^{P O+}$.

We propose that $V^{F B+}(G)=V^{P O+} / M$ and $g^{F B+}=g^{P O+}$. By rewriting (89), with $V^{F B+}(G)=V^{P O+} / M$ and $g^{F B+}=g^{P O+}$, we obtain

$$
M \frac{\partial F\left(g^{P O+}, G\right)}{\partial g}-V_{G}^{P O+}(G)(1-\alpha)-M \mu=0, \forall G, \quad \mu \geq 0 .
$$

We want to prove that $\bar{g}=g^{P O+}$ is the optimal quota of the above problem, i.e., there exists $\mu>0$ such that (91) holds. Using equations (87) and (91), we obtain:

$$
(M-1) \frac{\partial F\left(g^{P O+}, G\right)}{\partial g}-M \mu=0, \forall G, \quad \mu>0 .
$$

Thus, $\mu$ can be defined by:

$$
\mu=\frac{(M-1) \frac{\partial F\left(g^{P O+}, G\right)}{\partial g}}{M} .
$$

From (87), we know that

$$
\frac{\partial F\left(g^{P O+}, G\right)}{\partial g}>0
$$

then $\mu>0$, and $\bar{g}=g^{P O+}$.

In a similar way, using the Hamilton-Jacobi-Bellman equation in a finite time horizon with a scrap value, we can show that $g=g^{P O-}$ is a solution of the feedback problem with quota restriction $\bar{g}=g^{P O-}$ between 0 and $t_{a}$.

\section{References}

[1] de Frutos Cachorro J, Erdlenbruch K, Tidball M (2014) Optimal adaptation strategies to face shocks on groundwater resources. Journal of Economic Dynamics and Control 40: 134-153.

[2] Esteban E, Albiac J (2011) Groundwater and ecosystems damages: Questioning the Gisser-Sánchez effect. Ecological Economics 70: 2062-2069.

[3] Esteban E, Dinar A (2012) Groundwater-dependent ecosystems: How does the type if ecosystem affect the optimal management strategy. Working paper. 
[4] Gisser M, Sánchez DA (1980) Competition versus optimal control in groundwater pumping, Water Resources Research 31: 638-642.

[5] Koundouri P (2004) Current Issues in the Economics of Groundwater Resource Management. Journal of Economic Surveys 18(5): 703-740.

[6] López-Gunn E (2012) Groundwater governance and social capital. Geoforum 43: 11401151.

[7] López Sanz G (1993) El acuífero 23 de la Mancha Occidental y el acuífero 24 del Campo de Montiel : Funcionamiento, Gestión, problemática y alternativas. Paper presented at the Symposium on Water Management in Campo de Montiel and Western La Mancha, Alcázar de San Juan (Ciudad Real), 13 november 1993.

[8] Negri DH (1989) The Common Property resource as a Differential Game. Water Resources Research 25: 9-15.

[9] Nieswiadomy M (1988) Input Substitution in Irrigated Agriculture in the High Plains of Texas 1970-80. Western Journal of Agricultural Economics 13: 63-70.

[10] Olcina Cantos J (2001) Tipología de sequías en España. Ería 56: 201-227.

[11] Provencher B, Burt O (1993) The Externalities Associated with the Common Property Exploitation of Groundwater. Journal of Environmental Economics and Management 24: $139-158$.

[12] Rubio SJ, Casino B (2001) Competitive versus efficient extraction of a common property resource: The groundwater case. Journal of Economics Dynamics and Control 25: $1117-1137$.

[13] Rubio SJ, Casino B (2003) Strategic Behavior and Efficiency in the common Property Extraction of Groundwater. Environmental and Resource Economics 26: 73-87.

[14] Tsur Y, Zemel A (2004) Endangered aquifers: groundwater management under threats of catastrophic events. Water Resources Research 40: 1-10. 
[15] Tsur Y, Zemel A (2014) Dynamic and stochastic analysis of environmental and natural resources. In: Fischer MM, Nijkamp P (Eds) Handbook of Regional Science. Springer, pp. 929-949. 